\title{
On Space-Time Code Design with A Conditional PIC Group Decoding
}

\author{
Tianyi $\mathrm{Xu}$ and Xiang-Gen Xia, Fellow, IEEE
}

\begin{abstract}
Space-time code designs based on a partial interference cancellation (PIC) group decoding have been recently proposed. The PIC group decoding complexity depends on the group size and is between the lowest linear receiver complexity and the highest ML decoding complexity. The symbol rate for a space-time code achieving full diversity with the PIC group decoding is also between those for the linear receivers and the ML decoding. In this paper, we propose a new decoding, called conditional PIC group decoding, that is between the PIC group decoding and the ML decoding. With the proposed new decoding, we obtain a new design criterion for space-time codes to achieve full diversity, which is also between the one with the PIC group decoding and the one with the ML decoding. We then present some designs that satisfy the new criterion and in the meantime have higher symbol rates than that for the PIC group decoding.
\end{abstract}

Index Terms-full diversity, partial interference cancellation, group decoding, cyclotomic lattice design, space-time block codes.

\section{INTRODUCTION}

$\mathbf{F}$ ULL diversity achieving space-time code designs with low complexity decodings include orthogonal space-time codes (OSTC) [1]-[7], quasi OSTC type codes with simplified ML decoding [8]-[16], extended OSTC with conditional ML detection [17]-[20], codes with linear receivers [21]-[25], and codes with PIC group decoding [26], [28]. Due to the orthogonality constraint, the symbol rates for OSTC type codes decrease to $1 / 2$ [4] when the number of transmit antennas gets large. When the orthogonality is relaxed so that the full diversity can be achieved with linear receivers, the symbol rates can still be close to 1 when the number of transmit antennas gets large [23]. When the linear receiver is generalized/relaxed to the PIC group decoding, the symbol rates for full diversity achieving codes can be increased to be more than 1 but upper bounded by the group size [26]. It is known that the symbol rates for full diversity codes with the ML decoding can be full, i.e., $n_{t}$, for $n_{t}$ transmit antennas [29]-[33], including perfect codes [31], [32]. A natural question is whether there are full diversity achieving codes with symbol rates higher than that with the PIC group decoding, and with a decoding of a lower complexity than the ML decoding.

Motivated from the results in [17]-[20] on extended OSTC with conditional ML detection, in this paper we propose a

This work was supported in part by the National Science Foundation (NSF) under Grant CCF-0964500, the Air Force Office of Scientific Research (AFOSR) under Grant No. FA9550-08-1-0219, and the World Class University (WCU) Program, National Research Foundation, Korea.

The authors are with the Department of Electrical and Computer Engineering, University of Delaware, Newark, DE 19716, USA. Email: \{txu, xxia\}@ee.udel.edu. conditional PIC group decoding that can be stated as follows. For each trial of a fixed group of information symbols, the remaining information symbols are decoded with the PIC group decoding. The final decoding is the optimal solution among all the trials of the fixed group of symbols. It is clear that the complexity of this conditional PIC group decoding is between those of the PIC group decoding in [26] and the ML decoding. We obtain a new design criterion for space-time codes to achieve full diversity with the conditional PIC group decoding. The new criterion is also between those with the PIC group decoding and with the ML decoding. We then propose some designs that satisfy the new criterion, i.e., achieve full diversity with the conditional PIC group decoding, and in the meantime, their symbol rates are higher than those designed for the PIC group decoding in [26], [28].

This paper is organized as follows. In Section II, we describe the system model and the PIC group decoding. In Section III, we introduce the conditional PIC group decoding and present the new design criterion. In Section IV, we propose some code designs. In Section V, we show some simulation results.

\section{System Model AND PIC GROup DeCOding}

We consider an MIMO transmission with $n_{t}$ transmit and $n_{r}$ receive antennas over a quasi-static Rayleigh block-fading channel. The channel model is written as follows,

$$
\mathbf{Y}=\sqrt{\frac{\rho}{n_{t}}} \mathbf{X H}+\mathbf{N}
$$

where $\mathbf{Y} \in \mathbb{C}^{t \times n_{r}}$ is the received signal matrix in $t$ time slots, $\mathbf{X} \in \mathbb{C}^{t \times n_{t}}$ is a codeword matrix from a space-time block code (STBC), or simply $\mathbf{X}$ is an STBC, $\mathbf{H}=\left(h_{i j}\right)_{n_{t} \times n_{r}}$ is the $n_{t} \times n_{r}$ channel matrix whose entries are assumed i.i.d. with distribution $\mathcal{C N}(0,1), \mathbf{N} \in \mathbb{C}^{t \times n_{r}}$ is an additive white Gaussian noise matrix with i.i.d. entries $n_{i, j} \sim \mathcal{C N}(0,1)$ and $\rho$ is the average signal-to-noise-ratio at the receiver.

In this paper, for convenience we only consider that information symbols $s_{l}, l=1, \cdots, L$, are coded by linear dispersion STBC [35], [36] as

$$
\mathbf{X}=\mathbf{X}\left(s_{1}, \cdots, s_{L}\right)=\sum_{l=1}^{L} \mathbf{A}_{l} s_{l}
$$

where $\mathbf{A}_{l} \in \mathbb{C}^{t \times n_{t}}$ is a linear STBC weight matrix. When the complex conjugated information symbols are involved, they can be converted into the above form if their real and imaginary parts are split into two independent variables.

To decode the transmitted information symbol vector $\mathbf{s}=$ $\left[s_{1}, s_{2}, \cdots, s_{L}\right]^{T}$ at the receiver, the system model in (1) needs 
to be rewritten as

$$
\mathbf{y}=\sqrt{\rho} \mathbf{G s}+\mathbf{n},
$$

where $\mathbf{y} \in \mathbb{C}^{t n_{r}}$ is the received signal vector, $\mathbf{G} \in \mathbb{C}^{t n_{r} \times L}$ is an equivalent channel matrix [23], [26], $\mathbf{n} \in \mathbb{C}^{t n_{r}}$ is the additive white Gaussian noise vector.

We now describe the PIC group decoding algorithm studied in [26]. Define the index set $\mathcal{I}$ as $\mathcal{I}=\{1,2, \cdots, L\}$, where $L$ is the number of information symbols in $\mathbf{s}$. First we partition $\mathcal{I}$ into $N$ groups: $\mathcal{I}_{1}, \mathcal{I}_{2}, \cdots, \mathcal{I}_{N}$. Each index subset $\mathcal{I}_{k}$ can be written as follows,

$$
\mathcal{I}_{k}=\left\{i_{k, 1}, i_{k, 2}, \cdots, i_{k, n_{k}}\right\}, k=1,2, \cdots, N,
$$

where $n_{k} \triangleq\left|\mathcal{I}_{k}\right|$ is the cardinality of the subset $\mathcal{I}_{k}$. We call $\mathcal{I}=\left\{\mathcal{I}_{1}, \mathcal{I}_{2}, \cdots, \mathcal{I}_{N}\right\}$ a grouping scheme. For such a grouping scheme, we have the following two equations

$$
\mathcal{I}=\bigcup_{i=1}^{N} \mathcal{I}_{i} \quad \text { and } \quad \sum_{i=1}^{N} n_{i}=L .
$$

Define $\mathbf{s}_{\mathcal{I}_{k}}$ as the information symbol vector that contains the symbols with indices in $\mathcal{I}_{k}$, i.e.,

$$
\mathbf{s}_{\mathcal{I}_{k}}=\left[s_{i_{k, 1}}, s_{i_{k, 2}}, \cdots, s_{i_{k, n_{k}}}\right]^{T} .
$$

Let the column vectors of an equivalent channel matrix $\mathbf{G}$ be $\mathbf{g}_{1}, \mathbf{g}_{2}, \cdots, \mathbf{g}_{L}$. Similarly, we can define $\mathbf{G}_{\mathcal{I}_{k}}$ as

$$
\mathbf{G}_{\mathcal{I}_{k}}=\left[\mathbf{g}_{i_{k, 1}}, \mathbf{g}_{i_{k, 2}}, \cdots, \mathbf{g}_{i_{k, n_{k}}}\right] .
$$

In this case, the STBC $\mathbf{X}$ in (2) can be also written as $\mathbf{X}=$ $\mathbf{X}\left(\mathbf{s}_{\mathcal{I}_{1}}, \cdots, \mathbf{s}_{\mathcal{I}_{N}}\right)$. With these notations, equation (3) can be rewritten as

$$
\mathbf{y}=\sqrt{\rho} \sum_{i=1}^{N} \mathbf{G}_{\mathcal{I}_{i}} \mathbf{s}_{\mathcal{I}_{i}}+\mathbf{n} .
$$

Suppose we want to decode the symbols embedded in group $\mathbf{S}_{\mathcal{I}_{i}}$. The PIC group decoding first implements linear interference cancellation with a suitable choice of matrix $\mathbf{Q}_{\mathcal{I}_{i}}$ in order to completely eliminate the interferences from other groups [26], i.e., $\mathbf{Q}_{\mathcal{I}_{i}} \mathbf{G}_{\mathcal{I}_{j}}=0, \forall j \neq i$ and $i=1,2, \cdots, N$. To satisfy this, we can, for example, choose $\mathbf{Q}_{\mathcal{I}_{i}}$ as the following zero-forcing filter

$\mathbf{Q}_{\mathcal{I}_{i}}=\mathbf{I}_{t n_{r}}-\mathbf{G}_{\mathcal{I}_{i}}^{c}\left(\left(\mathbf{G}_{\mathcal{I}_{i}}^{c}\right)^{H} \mathbf{G}_{\mathcal{I}_{i}}^{c}\right)^{-1}\left(\mathbf{G}_{\mathcal{I}_{i}}^{c}\right)^{H}, \quad i=1,2, \cdots, N$,

when the following matrix has full column rank:

$$
\mathbf{G}_{\mathcal{I}_{i}}^{c}=\left[\mathbf{G}_{\mathcal{I}_{1}}, \cdots, \mathbf{G}_{\mathcal{I}_{i-1}}, \mathbf{G}_{\mathcal{I}_{i+1}}, \cdots, \mathbf{G}_{\mathcal{I}_{N}}\right] .
$$

Then we have

$$
\mathbf{z}_{\mathcal{I}_{i}}=\mathbf{Q}_{\mathcal{I}_{i}} \mathbf{y}=\sqrt{\rho} \mathbf{Q}_{\mathcal{I}_{i}} \mathbf{G}_{\mathcal{I}_{i}} \mathbf{s}_{\mathcal{I}_{i}}+\mathbf{Q}_{\mathcal{I}_{i}} \mathbf{n}, \quad i=1,2, \cdots, N
$$

The symbols in group $\mathbf{s}_{\mathcal{I}_{i}}$ can be decoded with the ML decoding as follows [26]:

$$
\hat{\mathbf{s}}_{\mathcal{I}_{i}}=\arg \min _{\mathbf{s}_{\mathcal{I}_{i}} \in \mathcal{A}^{n_{i}}}\left\|\mathbf{z}_{\mathcal{I}_{i}}-\sqrt{\rho} \mathbf{Q}_{\mathcal{I}_{i}} \mathbf{G}_{\mathcal{I}_{i}} \mathbf{s}_{\mathcal{I}_{i}}\right\|^{2} .
$$

where $\mathcal{A}$ is a signal constellation for the information symbols.

In [26], [27], an STBC design criterion was derived to achieve full diversity with the PIC group decoding, which can be stated as follows.
Theorem 2.1: For an STBC $\mathbf{X}$ with the PIC group decoding, the full diversity is achieved when the code $\mathbf{X}$ satisfies the full rank criterion, i.e., it achieves full diversity when the ML receiver is used; and for a fixed $k, 1 \leq k \leq N$, any nonzero linear combination over $\Delta \mathcal{A}$ of the vectors in the $k$ th group $\boldsymbol{G}_{\mathcal{I}_{k}}$ does not belong to the space linearly spanned by all the vectors in the remaining vector groups over the complex field, i.e., $\mathbb{V}_{\mathcal{I}_{k}}$ defined in (12) in [26], for any $\mathbf{H} \neq 0$.

Notice that in the PIC group decoding algorithm, we may use successive interference cancellation (SIC) strategy to aid the decoding process. We call the SIC-aided PIC group decoding as PIC-SIC group decoding [26]. The basic idea of this method is to remove the already-decoded symbols from the received signals to reduce the interferences. For a decoding order, for example, $\left(\mathbf{s}_{\mathcal{I}_{1}}, \mathbf{s}_{\mathcal{I}_{2}}, \cdots, \mathbf{s}_{\mathcal{I}_{N}}\right)$, first, we can decode symbol group $\mathbf{s}_{\mathcal{I}_{1}}$ by the PIC group decoding to obtain $\hat{\mathbf{s}}_{\mathcal{I}_{1}}$. Remove the components of the already-detected symbol group $\hat{\mathbf{s}}_{\mathcal{I}_{1}}$ from (4):

$$
\mathbf{y}_{1}=\mathbf{y}-\sqrt{\rho} \mathbf{G}_{\mathcal{I}_{1}} \hat{\mathbf{s}}_{\mathcal{I}_{1}} .
$$

Then, decode $\mathbf{s}_{\mathcal{I}_{2}}$ from (6) by the PIC group decoding. Repeat this process until all symbols are decoded. Then, the full diversity criterion for the PIC-SIC group decoding can be stated as follows [26], [27].

Theorem 2.2: For an STBC $\mathbf{X}$ with the PIC-SIC group decoding, the full diversity is achieved when the code $\mathbf{X}$ satisfies the full rank criterion, i.e., it achieves full diversity when the ML receiver is used; and at each decoding stage, for $\mathbf{G}_{\mathcal{I}_{k}}$, which corresponds to the current to-be decoded symbol group $\mathbf{s}_{\mathcal{I}_{k}}$, any nonzero linear combination over $\Delta \mathcal{A}$ of the vectors in $\boldsymbol{G}_{\mathcal{I}_{k}}$ does not belong to the space linearly spanned by all the vectors in the group $\left[\boldsymbol{G}_{\mathcal{I}_{k+1}}, \cdots, \boldsymbol{G}_{\mathcal{I}_{N}}\right]$ over the complex field for any $\mathbf{H} \neq 0$.

We next propose a new decoding algorithm called conditional PIC group decoding.

\section{Conditional PIC Group Decoding And A NeW DESIGN CRITERION}

Motivated from the conditional detection in [17]-[20] on the conditional fast ML decoding for orthogonal codes, we now propose a conditional PIC group decoding. Our proposed conditional PIC group decoding method is implemented in two major steps. First, estimate the information symbols in the first $N-1$ groups $\mathbf{s}_{\mathcal{I}_{1}}, \mathbf{s}_{\mathcal{I}_{2}}, \cdots, \mathbf{s}_{\mathcal{I}_{N-1}}$ using the PIC group decoding for every possible trial of the information symbols in the last group $\mathbf{s}_{\mathcal{I}_{N}}$ : for every $\overline{\mathbf{s}}_{\mathcal{I}_{N}} \in \mathcal{A}^{n_{N}}$, where $\mathcal{A}$ is the constellation used, cancel it from the received signal:

$\mathbf{y}-\sqrt{\rho} \mathbf{G}_{\mathcal{I}_{N}} \overline{\mathbf{s}}_{\mathcal{I}_{N}}=\sqrt{\rho} \sum_{i=1}^{N-1} \mathbf{G}_{\mathcal{I}_{i}} \mathbf{s}_{\mathcal{I}_{i}}+\mathbf{n}+\sqrt{\rho} \mathbf{G}_{\mathcal{I}_{N}}\left(\mathbf{s}_{\mathcal{I}_{N}}-\overline{\mathbf{s}}_{\mathcal{I}_{N}}\right)$.

Apply the PIC group decoding to decode $\mathbf{s}_{\mathcal{I}_{i}}, 1 \leq i \leq N-1$, from the above $\mathbf{y}-\sqrt{\rho} \mathbf{G}_{\mathcal{I}_{N}} \overline{\mathbf{s}}_{\mathcal{I}_{N}}$ for every $\overline{\mathbf{s}}_{\mathcal{I}_{N}}$, and denote the decoding results by $\mathbf{s}_{\mathcal{I}_{i}}^{\text {IC }}\left(\overline{\mathbf{s}}_{\mathcal{I}_{N}}\right)$, which are functions of $\overline{\mathbf{s}}_{\mathcal{I}_{N}}$. This first major step is named as the PIC group decoding step. Then, the second major step is to choose $\mathbf{s}_{\mathcal{I}_{N}}$ to minimize the 


$$
\mathbf{s}_{\mathcal{I}_{N}}^{\text {condPIC }}=\arg \min _{\overline{\mathbf{s}}_{\mathcal{I}_{N} \in \mathcal{A}^{n_{N}}}}\left|\mathbf{y}-\sqrt{\rho}\left(\sum_{i=1}^{N-1} \mathbf{G}_{\mathcal{I}_{i}} \mathbf{s}_{\mathcal{I}_{i}}^{P I C}\left(\overline{\mathbf{s}}_{\mathcal{I}_{N}}\right)+\mathbf{G}_{\mathcal{I}_{N}} \overline{\mathbf{s}}_{\mathcal{I}_{N}}\right)\right|^{2}
$$

ML metric from all the results in the first step as (8) above; and for $i=1,2, \cdots, N-1$,

$$
\mathbf{s}_{\mathcal{I}_{i}}^{\text {condPIC }}=\mathbf{s}_{\mathcal{I}_{i}}^{\text {PIC }}\left(\mathbf{s}_{\mathcal{I}_{N}}^{\text {condPIC }}\right) .
$$

This second major step is named as the ML step.

Suppose that $\mathcal{A}^{n_{N}}=\left\{a_{1}, a_{2}, \cdots, a_{q}\right\}$. Then, the conditional PIC group decoding algorithm can be described in details as:

1) Let $t=1$;

2) Let $\overline{\mathbf{s}}_{\mathcal{I}_{N}}=a_{t}$, cancel it from the received signal as (7);

3) Treat $\mathbf{n}+\sqrt{\rho} \mathbf{G}_{\mathcal{I}_{N}}\left(\mathbf{s}_{\mathcal{I}_{N}}-\overline{\mathbf{s}}_{\mathcal{I}_{N}}\right)$ as noise, and decode $\mathbf{s}_{\mathcal{I}_{1}}, \cdots, \mathbf{s}_{\mathcal{I}_{N-1}}$ using the PIC group decoding. The decoding results are $\mathbf{s}_{\mathcal{I}_{i}}^{\text {PIC }}\left(\overline{\mathbf{s}}_{\mathcal{I}_{N}}\right)$;

4) Calculate the ML metric

$$
\left|\mathbf{y}-\sqrt{\rho}\left(\sum_{i=1}^{N-1} \mathbf{G}_{\mathcal{I}_{i}} \mathbf{s}_{\mathcal{I}_{i}}^{P I C}\left(\overline{\mathbf{s}}_{\mathcal{I}_{N}}\right)+\mathbf{G}_{\mathcal{I}_{N}} \overline{\mathbf{s}}_{\mathcal{I}_{N}}\right)\right|^{2} ;
$$

5) If $t<q$, then set $t:=t+1$, go to Step 2; otherwise, go to Step 6;

6) Choose $\mathbf{s}_{\mathcal{I}_{N}}$ with the minimal ML metric in Step 4 , and the conditional PIC group decoding results are $\mathbf{s}_{\mathcal{I}_{1}}^{P I C}\left(\mathbf{s}_{\mathcal{I}_{N}}\right), \cdots, \mathbf{s}_{\mathcal{I}_{N-1}}^{P I C}\left(\mathbf{s}_{\mathcal{I}_{N}}\right), \mathbf{s}_{\mathcal{I}_{N}}$.

The decoding complexity of the PIC group decoding step is $O\left(|\mathcal{A}|^{c_{1}}\right)$, where $c_{1}$ is the maximum cardinality of $\mathcal{I}_{1}, \mathcal{I}_{2}, \cdots, \mathcal{I}_{N-1}$, i.e., $c_{1}=\max \left\{n_{1}, n_{2}, \cdots, n_{N-1}\right\}$. In the ML step, since we need to enumerate all possible trials of the group $\mathbf{s}_{\mathcal{I}_{N}}$, its complexity is $O\left(|\mathcal{A}|^{n_{N}}\right)$. Thus, the total decoding complexity is $O\left(|\mathcal{A}|^{c_{1}+n_{N}}\right)$. Comparing with the complexity, $O\left(|\mathcal{A}|^{n_{1}+n_{2}+\cdots+n_{N}}\right)$, of the ML decoding, the above complexity is much lower. Comparing with the PIC group decoding, the complexity of this conditional PIC group decoding method is higher. As shown in the following theorem, the full diversity criterion is weaker, which implies that we can design a higher rate code achieving the full diversity than that using the PIC group decoding. Our proposed method is also different from the PIC-SIC group decoding method. The PIC-SIC group decoding method cancels the interference from the already decoded symbol groups, but the proposed method, for any possible trial of a group of symbols, removes them from the received signal and decodes other symbol groups with the PIC group decoding, and then chooses the best solutions.

Theorem 3.1: For an STBC $\mathbf{X}$ with the above conditional PIC group decoding, the full diversity is achieved when the code $\mathbf{X}$ satisfies the full rank criterion, i.e., it achieves full diversity when the ML receiver is used; and for a fixed $k$, $1 \leq k \leq N-1$, any nonzero linear combination over $\Delta \mathcal{A}$ of the vectors in the $k$ th group $\boldsymbol{G}_{\mathcal{I}_{k}}$ does not belong to the space linearly spanned by all the vectors in the vector group $\left[\boldsymbol{G}_{\mathcal{I}_{1}}, \cdots, \boldsymbol{G}_{\mathcal{I}_{k-1}}, \boldsymbol{G}_{\mathcal{I}_{k+1}}, \cdots, \boldsymbol{G}_{\mathcal{I}_{N-1}}\right]$ over the complex field for any $\mathbf{H} \neq 0$, where a nonzero linear combination over $\Delta \mathcal{A}$ means that all the coefficients in the linear combination are taken from $\Delta \mathcal{A}$ and not all of them are zero.
Proof: Suppose information symbol vectors $\mathbf{s}_{\mathcal{I}_{i}}^{0}, i=$ $1,2, \cdots, N$, are transmitted. Then, there are two types of errors as follows. The first type of errors appear in the PIC group decoding step when we set $\overline{\mathbf{s}}_{\mathcal{I}_{N}}=\mathbf{s}_{\mathcal{I}_{N}}^{0}$. In this case, the equation (7) becomes

$$
\mathbf{y}^{\prime} \equiv \mathbf{y}-\sqrt{\rho} \mathbf{G}_{\mathcal{I}_{N}} \overline{\mathbf{s}}_{\mathcal{I}_{N}}=\sqrt{\rho} \sum_{i=1}^{N-1} \mathbf{G}_{\mathcal{I}_{i}} \mathbf{s}_{\mathcal{I}_{i}}^{0}+\mathbf{n}
$$

Since the conditions in Theorem 2.1 are satisfied by $\mathbf{G}_{\mathcal{I}_{1}}, \cdots, \mathbf{G}_{\mathcal{I}_{N-1}}$, the pairwise error probability of the detection of $\mathbf{s}_{\mathcal{I}_{i}}, i=1,2, \cdots, N-1$, when $\overline{\mathbf{s}}_{\mathcal{I}_{N}}=\mathbf{s}_{\mathcal{I}_{N}}^{0}$ is given, is upper bounded by (10) in the next page, for a positive constant $C_{1}$ and $\left(\mathbf{s}_{\mathcal{I}_{1}}^{0}, \mathbf{s}_{\mathcal{I}_{2}}^{0}, \cdots, \mathbf{s}_{\mathcal{I}_{N-1}}^{0}\right) \neq$ $\left(\mathbf{s}_{\mathcal{I}_{1}}^{\text {IC }}\left(\mathbf{s}_{\mathcal{I}_{N}}^{0}\right), \mathbf{s}_{\mathcal{I}_{2}}^{\text {IIC }}\left(\mathbf{s}_{\mathcal{I}_{N}}^{0}\right), \cdots, \mathbf{s}_{\mathcal{I}_{N-1}}^{\text {IIC }}\left(\mathbf{s}_{\mathcal{I}_{N}}^{0}\right)\right)$.

The second type of errors appear in the ML step of the decoding, but in the PIC group decoding step, when we set $\overline{\mathbf{s}}_{\mathcal{I}_{N}}=\mathbf{s}_{\mathcal{I}_{N}}^{0}$, the detection results are correct, i.e., $\mathbf{s}_{\mathcal{I}_{i}}^{P I C}\left(\mathbf{s}_{\mathcal{I}_{N}}^{0}\right)=$ $\mathbf{s}_{\mathcal{I}_{i}}^{0}$ for $i=1,2, \cdots, N-1$. In this step, for any $\overline{\mathbf{s}}_{\mathcal{I}_{N}}$, from (7) we obtain $\mathbf{s}_{\mathcal{I}_{i}}^{P I C}\left(\overline{\mathbf{s}}_{\mathcal{I}_{N}}\right), i=1,2, \cdots, N-1$. Define set

$$
\mathcal{A}^{\prime}=\left\{\left(\mathbf{s}_{\mathcal{I}_{1}}^{\text {IC }}\left(\overline{\mathbf{s}}_{\mathcal{I}_{N}}\right), \cdots, \mathbf{s}_{\mathcal{I}_{N-1}}^{\text {IC }}\left(\overline{\mathbf{s}}_{\mathcal{I}_{N}}\right), \overline{\mathbf{s}}_{\mathcal{I}_{N}}\right) \mid \overline{\mathbf{s}}_{\mathcal{I}_{N}} \in \mathcal{A}^{n_{N}}\right\} .
$$

Due to the above error pattern, the above set $\mathcal{A}^{\prime}$ contains the truely transmitted $\mathbf{s}_{\mathcal{I}_{i}}^{0}$ for $i=1,2, \cdots, N$, i.e., $\left(\mathbf{s}_{\mathcal{I}_{1}}^{0}, \mathbf{s}_{\mathcal{I}_{2}}^{0}, \cdots, \mathbf{s}_{\mathcal{I}_{N}}^{0}\right) \in \mathcal{A}^{\prime}$.

The detection of the remaining symbols can be processed as (11) in the next page, where $\|\cdot\|_{F}$ is the Frobenius norm of a matrix. Let $\hat{\mathbf{s}}_{\mathcal{I}_{i}}=\mathbf{s}_{\mathcal{I}_{i}}^{P I C}\left(\hat{\mathbf{s}}_{\mathcal{I}_{N}}\right)$ for $i=1,2, \cdots, N-1$. It is easy to see that $\left(\hat{\mathbf{s}}_{\mathcal{I}_{1}}, \hat{\mathbf{s}}_{\mathcal{I}_{2}}, \cdots, \hat{\mathbf{s}}_{\mathcal{I}_{N}}\right) \in \mathcal{A}^{\prime}$ and it is the solution of the ML decoding over $\mathcal{A}^{\prime}$.

Since $\mathbf{X}$ satisfies the full rank criterion on $\mathcal{A}^{L}$, it is obvious that $\mathbf{X}$ satisfies the full rank criterion on $\mathcal{A}^{\prime}$ that is a subset of $\mathcal{A}^{L}$. Thus, the pairwise error probability in the ML step above can be bounded by

$\operatorname{Pr}\left(\left[\mathbf{s}_{\mathcal{I}_{1}}^{0}, \mathbf{s}_{\mathcal{I}_{2}}^{0}, \cdots, \mathbf{s}_{\mathcal{I}_{N}}^{0}\right] \stackrel{\mathrm{ML}}{\longrightarrow}\left[\hat{\mathbf{s}}_{\mathcal{I}_{1}}, \hat{\mathbf{s}}_{\mathcal{I}_{2}}, \cdots, \hat{\mathbf{s}}_{\mathcal{I}_{N}}\right]\right) \leq C_{2} \rho^{-n_{t} n_{r}}$

for a positive constant $C_{2}$ and $\left(\mathbf{s}_{\mathcal{I}_{1}}^{0}, \mathbf{s}_{\mathcal{I}_{2}}^{0}, \cdots, \mathbf{s}_{\mathcal{I}_{N}}^{0}\right) \neq$ $\left(\hat{\mathbf{s}}_{\mathcal{I}_{1}}, \hat{\mathbf{s}}_{\mathcal{I}_{2}}, \cdots, \hat{\mathbf{s}}_{\mathcal{I}_{N}}\right)$.

The total pairwise error probability for the proposed decoding method,

$$
\operatorname{Pr}\left(\left[\mathbf{s}_{\mathcal{I}_{1}}^{0}, \mathbf{s}_{\mathcal{I}_{2}}^{0}, \cdots, \mathbf{s}_{\mathcal{I}_{N}}^{0}\right] \longrightarrow\left[\mathbf{s}_{\mathcal{I}_{1}}^{\prime}, \mathbf{s}_{\mathcal{I}_{2}}^{\prime}, \cdots, \mathbf{s}_{\mathcal{I}_{N}}^{\prime}\right]\right)
$$

for $\left(\mathbf{s}_{\mathcal{I}_{1}}^{0}, \mathbf{s}_{\mathcal{I}_{2}}^{0}, \cdots, \mathbf{s}_{\mathcal{I}_{N}}^{0}\right) \neq\left(\mathbf{s}_{\mathcal{I}_{1}}^{\prime}, \mathbf{s}_{\mathcal{I}_{2}}^{\prime}, \cdots, \mathbf{s}_{\mathcal{I}_{N}}^{\prime}\right)$, can be expressed in two parts. One is that when in the PIC group decoding step, setting $\overline{\mathbf{s}}_{\mathcal{I}_{N}}=\mathbf{s}_{\mathcal{I}_{N}}^{0}$, the detection of $\mathbf{s}_{\mathcal{I}_{1}}, \mathbf{s}_{\mathcal{I}_{2}}, \cdots, \mathbf{s}_{\mathcal{I}_{N-1}}$ has no error, i.e., the truely transmitted symbol $\left(\mathbf{s}_{\mathcal{I}_{1}}, \mathbf{s}_{\mathcal{I}_{1}}, \cdots, \mathbf{s}_{\mathcal{I}_{N}}\right)$ is in the set $\mathcal{A}^{\prime}$. And in the ML step, we decode the symbols erroneously to $\left[\mathbf{s}_{\mathcal{I}_{1}}^{\prime}, \mathbf{s}_{\mathcal{I}_{2}}^{\prime}, \cdots, \mathbf{s}_{\mathcal{I}_{N}}^{\prime}\right]$. This part is due to the second type of errors. The other is that error occurs in the PIC group decoding 


$$
\operatorname{Pr}\left(\left[\mathbf{s}_{\mathcal{I}_{1}}^{0}, \mathbf{s}_{\mathcal{I}_{2}}^{0}, \cdots, \mathbf{s}_{\mathcal{I}_{N-1}}^{0} \mid \overline{\mathbf{s}}_{\mathcal{I}_{N}}=\mathbf{s}_{\mathcal{I}_{N}}^{0} \stackrel{\text { PIC group }}{\longrightarrow}\left[\mathbf{s}_{\mathcal{I}_{1}}^{P I C}\left(\mathbf{s}_{\mathcal{I}_{N}}^{0}\right), \mathbf{s}_{\mathcal{I}_{2}}^{P I C}\left(\mathbf{s}_{\mathcal{I}_{N}}^{0}\right), \cdots, \mathbf{s}_{\mathcal{I}_{N-1}}^{P I C}\left(\mathbf{s}_{\mathcal{I}_{N}}^{0}\right) \mid \overline{\mathbf{s}}_{\mathcal{I}_{N}}=\mathbf{s}_{\mathcal{I}_{N}}^{0}\right]\right) \leq C_{1} \rho^{-n_{t} n_{r}}\right.
$$

$$
\begin{aligned}
\hat{\mathbf{s}}_{\mathcal{I}_{N}} & =\arg \min _{\mathbf{s}_{\mathcal{I}_{N}} \in \mathcal{A}^{n} N}\left|\mathbf{y}-\sqrt{\rho}\left(\sum_{i=1}^{N-1} \mathbf{G}_{\mathcal{I}_{i}} \mathbf{s}_{\mathcal{I}_{i}}^{P I C}\left(\mathbf{s}_{\mathcal{I}_{N}}\right)+\mathbf{G}_{\mathcal{I}_{N}} \mathbf{s}_{\mathcal{I}_{N}}\right)\right|^{2} \\
& =\arg \min _{\left(\mathbf{s}_{\mathcal{I}_{1}}, \cdots, \mathbf{s}_{\mathcal{I}_{N}}\right) \in \mathcal{A}^{\prime}}\left|\mathbf{y}-\sqrt{\rho}\left(\sum_{i=1}^{N-1} \mathbf{G}_{\mathcal{I}_{i}} \mathbf{s}_{\mathcal{I}_{i}}+\mathbf{G}_{\mathcal{I}_{N}} \mathbf{s}_{\mathcal{I}_{N}}\right)\right|^{2} \\
& =\arg \min _{\left(\mathbf{s}_{\mathcal{I}_{1}}, \cdots, \mathbf{s}_{\mathcal{I}_{N}}\right) \in \mathcal{A}^{\prime}}\left\|\mathbf{Y}-\sqrt{\frac{\rho}{n_{t}}} \mathbf{X}\left(\mathbf{s}_{\mathcal{I}_{1}}, \cdots, \mathbf{s}_{\mathcal{I}_{N-1}}, \mathbf{s}_{\mathcal{I}_{N}}\right) \mathbf{H}\right\|_{F}^{2}
\end{aligned}
$$

step, when $\overline{\mathbf{s}}_{\mathcal{I}_{N}}=\mathbf{s}_{\mathcal{I}_{N}}^{0}$ is given. This part is due to the first type of errors.

For the first part, the pairwise error probability can be expressed as

$$
\begin{aligned}
& P_{1}=\operatorname{Pr}\left([ \mathbf { s } _ { \mathcal { I } _ { 1 } } ^ { 0 } , \mathbf { s } _ { \mathcal { I } _ { 2 } } ^ { 0 } , \cdots , \mathbf { s } _ { \mathcal { I } _ { N } } ^ { 0 } ] \stackrel { \text { PIC group } } { \longrightarrow } \left[\mathbf{s}_{\mathcal{I}_{1}}^{0},\right.\right. \\
& \left.\left.\mathbf{s}_{\mathcal{I}_{2}}^{0}, \cdots, \mathbf{s}_{\mathcal{I}_{N-1}}^{0} \mid \overline{\mathbf{s}}_{\mathcal{I}_{N}}=\mathbf{s}_{\mathcal{I}_{N}}^{0}\right] \stackrel{\text { ML }}{\longrightarrow}\left[\mathbf{s}_{\mathcal{I}_{1}}^{\prime}, \mathbf{s}_{\mathcal{I}_{2}}^{\prime}, \cdots, \mathbf{s}_{\mathcal{I}_{N}}^{\prime}\right]\right),
\end{aligned}
$$

which means that in the PIC group decoding step, with $\overline{\mathbf{s}}_{\mathcal{I}_{N}}=$ $\mathbf{s}_{\mathcal{I}_{N}}^{0}$, the detection result is $\left(\mathbf{s}_{\mathcal{I}_{1}}^{0}, \mathbf{s}_{\mathcal{I}_{2}}^{0}, \cdots, \mathbf{s}_{\mathcal{I}_{N-1}}^{0}\right)$, and, then, in the ML step, we decode the symbols to $\left(\mathbf{s}_{\mathcal{I}_{1}}^{\prime}, \mathbf{s}_{\mathcal{I}_{2}}^{\prime}, \cdots, \mathbf{s}_{\mathcal{I}_{N}}^{\prime}\right)$. Thus, we have

$$
\begin{aligned}
P_{1}= & \operatorname{Pr}\left(\left[\mathbf{s}_{\mathcal{I}_{1}}^{0}, \mathbf{s}_{\mathcal{I}_{2}}^{0}, \cdots, \mathbf{s}_{\mathcal{I}_{N-1}}^{0} \mid \mathbf{s}_{\mathcal{I}_{N}}=\mathbf{s}_{\mathcal{I}_{N}}^{0}\right] \stackrel{\text { PIC group }}{\longrightarrow}\right. \\
& {\left.\left[\mathbf{s}_{\mathcal{I}_{1}}^{0}, \mathbf{s}_{\mathcal{I}_{2}}^{0}, \cdots, \mathbf{s}_{\mathcal{I}_{N-1}}^{0} \mid \mathbf{s}_{\mathcal{I}_{N}}=\mathbf{s}_{\mathcal{I}_{N}}^{0}\right]\right) } \\
& \cdot \operatorname{Pr}\left(\left[\mathbf{s}_{\mathcal{I}_{1}}^{0}, \mathbf{s}_{\mathcal{I}_{2}}^{0}, \cdots, \mathbf{s}_{\mathcal{I}_{N-1}}^{0}, \mathbf{s}_{\mathcal{I}_{N}}^{0}\right] \stackrel{\mathrm{ML}}{\longrightarrow}\right. \\
& {\left.\left[\mathbf{s}_{\mathcal{I}_{1}}^{\prime}, \mathbf{s}_{\mathcal{I}_{2}}^{\prime}, \cdots, \mathbf{s}_{\mathcal{I}_{N}}^{\prime}\right]\right) } \\
\leq & \operatorname{Pr}\left(\left[\mathbf{s}_{\mathcal{I}_{1}}^{0}, \mathbf{s}_{\mathcal{I}_{2}}^{0}, \cdots, \mathbf{s}_{\mathcal{I}_{N-1}}^{0}, \mathbf{s}_{\mathcal{I}_{N}}^{0}\right] \stackrel{\mathrm{ML}}{\longrightarrow}\right. \\
\leq & {\left.\left[\mathbf{s}_{\mathcal{I}_{1}}^{\prime}, \mathbf{s}_{\mathcal{I}_{2}}^{\prime}, \cdots, \mathbf{s}_{\mathcal{I}_{N}}^{\prime}\right]\right) } \\
\leq & C_{2} \rho^{-n_{t} n_{r}} .
\end{aligned}
$$

where the last inequality comes from (12).

The second part of the pairwise error probability can be written as (13) in the next page. Each component in (13) can be bounded as (14) in the next page, where the last inequality comes from (10). Thus,

$$
\begin{aligned}
P_{2} \leq & \sum_{\substack{\left(\mathbf{s}_{\mathcal{I}_{1}}^{\prime}, \mathbf{s}_{\mathcal{I}_{2}}^{\prime \prime}, \cdots, \mathbf{s}_{\mathcal{I}_{N-1}}^{\prime \prime}\right) \in \mathcal{A}^{L-n_{N}},\left(\mathbf{s}_{\mathcal{I}_{1}}^{\prime \prime} \mathbf{s}_{\mathcal{I}_{2}}^{\prime \prime}, \cdots, \mathbf{s}_{\mathcal{I}_{N-1}}^{\prime \prime} \neq\left(\mathbf{s}_{\mathcal{I}_{1}}^{0}, \mathbf{s}_{\mathcal{I}_{2}}^{0}, \cdots, \mathbf{s}_{\mathcal{I}_{N-1}}^{0}\right)\right.}} C_{1} \rho^{-n_{t} n_{r}} \\
= & \left(|\mathcal{A}|^{l-n_{N}}-1\right) C_{1} \rho^{-n_{t} n_{r}} .
\end{aligned}
$$

Based on these two parts, we have the pairwise error probability of the conditional PIC group decoding as

$$
\begin{aligned}
& \operatorname{Pr}\left(\left[\mathbf{s}_{\mathcal{I}_{1}}^{0}, \mathbf{s}_{\mathcal{I}_{2}}^{0}, \cdots, \mathbf{s}_{\mathcal{I}_{N}}^{0}\right] \longrightarrow\left[\mathbf{s}_{\mathcal{I}_{1}}^{\prime}, \mathbf{s}_{\mathcal{I}_{2}}^{\prime}, \cdots, \mathbf{s}_{\mathcal{I}_{N}}^{\prime}\right]\right) \\
= & P_{1}+P_{2} \\
\leq & \left(C_{2}+\left(|\mathcal{A}|^{l-n_{N}}-1\right) C_{1}\right) \rho^{-n_{t} n_{r}} .
\end{aligned}
$$

This proves that the code can achieve full diversity with the proposed decoding method.

We similarly propose a conditional PIC-SIC group decoding by replacing the PIC group decoding with the PIC-SIC group decoding [26] in the first step. We have the following theorem, which can be similarly proved.

Theorem 3.2: For an STBC $\mathbf{X}$ with the conditional PIC-SIC group decoding, the full diversity is achieved when the code $\mathbf{X}$ satisfies the full rank criterion, i.e., it achieves full diversity when the ML decoding is used; and at each decoding stage, for $\mathbf{G}_{\mathcal{I}_{k}}$, which corresponds to the current to-be decoded symbol group $\mathbf{s}_{\mathcal{I}_{k}}$, any nonzero linear combination over $\Delta \mathcal{A}$ of the vectors in $\boldsymbol{G}_{\mathcal{I}_{k}}$ does not belong to the space linearly spanned by all the vectors in the group $\left[\boldsymbol{G}_{\mathcal{I}_{k+1}}, \cdots, \boldsymbol{G}_{\mathcal{I}_{N-1}}\right]$ over the complex field for any $\mathbf{H} \neq 0$.

The full diversity criteria in Theorems 3.1 and 3.2 only require a kind of linear independences (the second condition) of the first $N-1$ vector groups, which is weaker than the requirement of the same kind of linear independences (the second condition) of all the $N$ vector groups in Theorems 2.1 and 2.2 [26], [27]. With this reduced requirement in the criteria, we may add more symbol groups with the same number of time slots using the conditional PIC (or PIC-SIC) group decoding to achieve full diversity, as we shall see in next section. Thus, it is possible to design a higher rate full diversity code than using the PIC (or PIC-SIC) group decoding.

Example 1: Consider the full rate $2 \times 2$ STBC proposed in [17]:

$$
\mathbf{X}=\left[\begin{array}{cc}
a s_{1}+b s_{3} & a s_{2}+b s_{4} \\
-c s_{2}^{*}-d s_{4}^{*} & c s_{1}^{*}+d s_{3}^{*}
\end{array}\right]
$$

where $\left(s_{1}, s_{2}, s_{3}, s_{4}\right)$ are information symbols and the star stands for the complex conjugate, $a, b, c$ and $d$ are complexvalued design parameters with the same magnitude $1 / \sqrt{2}$, i.e., $|a|=|b|=|c|=|d|=1 / \sqrt{2}$. Suppose we use one receive antenna. The equivalent channel matrix $\mathbf{G}$ can be written as

$$
\begin{aligned}
\mathbf{G} & =\frac{1}{\sqrt{2}}\left[\mathbf{g}_{1}, \mathbf{g}_{2}, \mathbf{g}_{3}, \mathbf{g}_{4}\right] \\
& =\frac{1}{\sqrt{2}}\left[\begin{array}{cccc}
a h_{1} & a h_{2} & b h_{1} & b h_{2} \\
c^{*} h_{2}^{*} & -c^{*} h_{1}^{*} & d^{*} h_{2}^{*} & -d^{*} h_{1}^{*}
\end{array}\right] .
\end{aligned}
$$

It is easy to verify that $\mathbf{g}_{1} \perp \mathbf{g}_{2}$, and, consequently, $\mathbf{g}_{1}$ and $\mathbf{g}_{2}$ are linearly independent and thus satisfy the second condition 


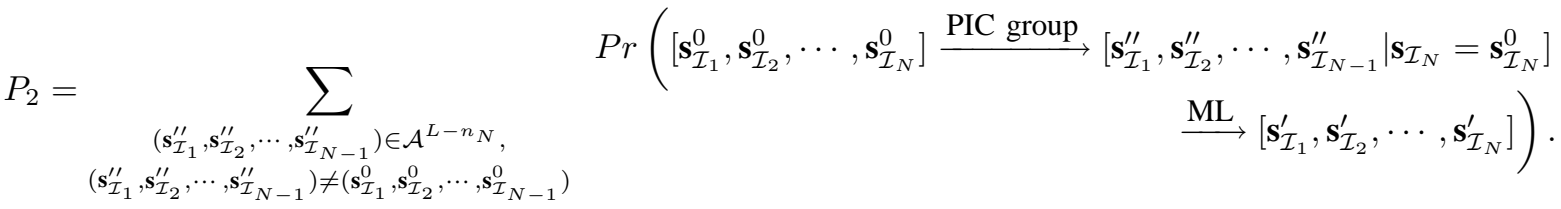

$$
\begin{aligned}
& \operatorname{Pr}\left(\left[\mathbf{s}_{\mathcal{I}_{1}}^{0}, \mathbf{s}_{\mathcal{I}_{2}}^{0}, \cdots, \mathbf{s}_{\mathcal{I}_{N}}^{0}\right] \stackrel{\text { PIC group }}{\longrightarrow}\left[\mathbf{s}_{\mathcal{I}_{1}}^{\prime \prime}, \mathbf{s}_{\mathcal{I}_{2}}^{\prime \prime}, \cdots, \mathbf{s}_{\mathcal{I}_{N-1}}^{\prime \prime} \mid \mathbf{s}_{\mathcal{I}_{N}}=\mathbf{s}_{\mathcal{I}_{N}}^{0}\right] \stackrel{\text { ML }}{\longrightarrow}\left[\mathbf{s}_{\mathcal{I}_{1}}^{\prime}, \mathbf{s}_{\mathcal{I}_{2}}^{\prime}, \cdots, \mathbf{s}_{\mathcal{I}_{N}}^{\prime}\right]\right) \\
& =\operatorname{Pr}\left(\left[\mathbf{s}_{\mathcal{I}_{1}}^{0}, \mathbf{s}_{\mathcal{I}_{2}}^{0}, \cdots, \mathbf{s}_{\mathcal{I}_{N-1}}^{0} \mid \mathbf{s}_{\mathcal{I}_{N}}=\mathbf{s}_{\mathcal{I}_{N}}^{0}\right] \stackrel{\text { PIC group }}{\longrightarrow}\left[\mathbf{s}_{\mathcal{I}_{1}}^{\prime \prime}, \mathbf{s}_{\mathcal{I}_{2}}^{\prime \prime}, \cdots, \mathbf{s}_{\mathcal{I}_{N-1}}^{\prime \prime} \mid \mathbf{s}_{\mathcal{I}_{N}}=\mathbf{s}_{\mathcal{I}_{N}}^{0}\right]\right) \\
& \cdot \operatorname{Pr}\left(\left[\mathbf{s}_{\mathcal{I}_{1}}^{\prime \prime}, \mathbf{s}_{\mathcal{I}_{2}}^{\prime \prime}, \cdots, \mathbf{s}_{\mathcal{I}_{N-1}}^{\prime \prime}, \mathbf{s}_{\mathcal{I}_{N}}^{0}\right] \stackrel{\mathrm{ML}}{\longrightarrow}\left[\mathbf{s}_{\mathcal{I}_{1}}^{\prime}, \mathbf{s}_{\mathcal{I}_{2}}^{\prime}, \cdots, \mathbf{s}_{\mathcal{I}_{N}}^{\prime}\right]\right) \\
& \leq \operatorname{Pr}\left(\left[\mathbf{s}_{\mathcal{I}_{1}}^{0}, \mathbf{s}_{\mathcal{I}_{2}}^{0}, \cdots, \mathbf{s}_{\mathcal{I}_{N-1}}^{0} \mid \mathbf{s}_{\mathcal{I}_{N}}=\mathbf{s}_{\mathcal{I}_{N}}^{0}\right] \stackrel{\text { PIC group }}{\longrightarrow}\left[\mathbf{s}_{\mathcal{I}_{1}}^{\prime \prime}, \mathbf{s}_{\mathcal{I}_{2}}^{\prime \prime}, \cdots, \mathbf{s}_{\mathcal{I}_{N-1}}^{\prime \prime} \mid \mathbf{s}_{\mathcal{I}_{N}}=\mathbf{s}_{\mathcal{I}_{N}}^{0}\right]\right) \\
& \leq C_{1} \rho^{-n_{t} n_{r}},
\end{aligned}
$$

in Theorem 3.1. Therefore, with the grouping scheme $\mathcal{I}_{1}=$ $\{1\}, \mathcal{I}_{2}=\{2\}$ and $\mathcal{I}_{3}=\{3,4\}$, we can decode this code by the conditional PIC group decoding. This algorithm is then the same as the one proposed in [17].

Before the ending of this section, we have a remark. Regarding to the decoding complexity for the conditional PIC group decoding, we may use sphere decoding [37][43] to reduce the complexity. We only need to search the information symbols in the last group, $\mathbf{s}_{\mathcal{I}_{N}}$, in a hypersphere around the received signal, not every possible trials in $\mathcal{A}^{n_{N}}$. Using the sphere decoding method in the ML step similar to [19], the decoding complexity is much lower than before. Moreover, in the PIC group decoding step, we can also use sphere decoding in each group instead of the ML decoding in (5). For an MIMO system with independent and identify distributed channel coefficients, it has been shown that the average complexity of sphere decoding is in polynomial order, roughly cubic, of the number of unknown variables, for a wide range of SNR and a moderate number of antennas and constellation sizes, although the exact complexity depends on the channels [41], [42]. Detailed complexity analysis for our proposed conditional PIC group decoding combined with the sphere decoding can be similarly done as [41], [42]. In Section $\mathrm{V}$, we show some simulation performances of the conditional PIC group decoding combined with the sphere decoding, which are similar to the performances of the conditional PIC group decoding.

\section{New CODE DESIGNS}

Let us first consider 2 transmit antennas. The proposed code is (17) in the next page, where $\theta=\frac{1}{2} \arctan 2$ [26], $\mathbf{i}=\sqrt{-1}$, and $s_{1}, s_{2}, \cdots, s_{6}$ are information symbols chosen from a QAM constellation $\mathcal{A}$. This code is transmitted over 3 time slots and has a symbol rate of 2. For convenience, we only consider the case of one receive antenna. According to the codeword structure, we can calculate the equivalent channel matrix (18) in the next page, where $h_{1}$ and $h_{2}$ are channel coefficients. It is not difficult to see that this code does not satisfy the criterion for the PIC group decoding with the group scheme $\mathcal{I}_{1}=\{1,2\}, \mathcal{I}_{2}=\{3,4\}$ and $\mathcal{I}_{3}=\{5,6\}$. Actually, for every group, any vector in this group can be linearly expressed by the vectors in the other two groups. Thus, this code does not satisfy the criterion to achieve full diversity with the PIC group decoding in [26], [27].

Suppose the conditional PIC group decoding is used to decode the code (17): first, estimate $s_{1}, s_{2}, s_{3}, s_{4}$ using the PIC group decoding for every possible trial of $\left(s_{5}, s_{6}\right)$; then, choose $\left(s_{5}, s_{6}\right)$ to minimize the ML metric from all results in the first step. Since $\tan \theta$ is an irrational number, we have $a_{1} h_{1} \cos \theta+a_{2} h_{1} \sin \theta \neq 0$ for $h_{1} \neq 0$ and any $a_{1}, a_{2} \in \mathbb{Z}[\mathbf{i}]$, where $\mathbb{Z}[\mathbf{i}]$ is the number ring generated by the integer ring $\mathbb{Z}$ and i. Similarly, $-b_{1} h_{2} \sin \theta+b_{2} h_{2} \cos \theta \neq 0$ for $h_{2} \neq 0$ and any $b_{1}, b_{2} \in \mathbb{Z}[\mathbf{i}]$. This implies that for $h_{1}$ and $h_{2}$ are not all 0 , any nonzero linear combination over $\mathbb{Z}[\mathbf{i}]$ of vectors from $\mathbf{G}_{\mathcal{I}_{1}}$ does not belong to the space linearly spanned by all the vectors from $\mathbf{G}_{\mathcal{I}_{2}}$ over the complex field, since any complex linear combination of the first components of the vectors in $\mathbf{G}_{\mathcal{I}_{2}}$ is always 0 and any complex linear combination of the second components of the vectors in $\mathbf{G}_{\mathcal{I}_{2}}$ is always 0 if $h_{1}=0$. This conclusion similarly holds when the order of $\mathbf{G}_{\mathcal{I}_{1}}$ and $\mathbf{G}_{\mathcal{I}_{2}}$ is switched. Since $\Delta \mathcal{A}$ is a subset of $\mathbb{Z}[\mathbf{i}]$, we have proved that, for $\mathbf{G}_{\mathcal{I}_{1}}$ and $\mathbf{G}_{\mathcal{I}_{2}}$, any nonzero linear combination over $\Delta \mathcal{A}$ of vectors from one vector group can not be linearly expressed by the vectors in the other vector group over the complex field. The following property can guarantee that this code has full rank property when a QAM constellation is used. Thus, from the new criterion we obtained in Theorem 3.1, this code can achieve full diversity with a QAM constellation and the conditional PIC group decoding.

Property 4.1: The matrix $\mathbf{X}$ in (17) has full rank when $s_{i} \in$ $\mathbb{Z}[\mathbf{i}]$ and at least one of $s_{i}$ is not 0 , where $\mathbb{Z}[\mathbf{i}]$ is the number ring generated by the integer ring $\mathbb{Z}$ and $\mathbf{i}$.

Proof: To prove this property, we need to verify that at least one of the $2 \times 2$ minors of matrix $\mathbf{X}$ is not 0 . 


$$
\begin{aligned}
& \mathbf{X}=\left[\begin{array}{cc}
s_{1} \cos \theta+s_{2} \sin \theta & e^{-\mathbf{i} \pi / 4}\left(-s_{5} \sin \theta+s_{6} \cos \theta\right) \\
s_{3} \cos \theta+s_{4} \sin \theta & -s_{1} \sin \theta+s_{2} \cos \theta \\
e^{-\mathbf{i} \pi / 4}\left(s_{5} \cos \theta+s_{6} \sin \theta\right) & -s_{3} \sin \theta+s_{4} \cos \theta
\end{array}\right] \\
& \mathbf{G}=\frac{1}{\sqrt{2}}\left[\begin{array}{cccccc}
h_{1} \cos \theta & h_{1} \sin \theta & 0 & 0 & -e^{-\mathbf{i} \pi / 4} h_{2} \sin \theta & e^{-\mathbf{i} \pi / 4} h_{2} \cos \theta \\
-h_{2} \sin \theta & h_{2} \cos \theta & h_{1} \cos \theta & h_{1} \sin \theta & 0 & 0 \\
0 & 0 & -h_{2} \sin \theta & h_{2} \cos \theta & e^{-\mathbf{i} \pi / 4} h_{1} \cos \theta & e^{-\mathbf{i} \pi / 4} h_{1} \sin \theta
\end{array}\right]
\end{aligned}
$$

Suppose all the $2 \times 2$ minors of matrix $\mathbf{X}$ are 0 . For example,

$\operatorname{det}\left(\left[\begin{array}{cc}s_{1} \cos \theta+s_{2} \sin \theta & e^{-\mathbf{i} \pi / 4}\left(-s_{5} \sin \theta+s_{6} \cos \theta\right) \\ s_{3} \cos \theta+s_{4} \sin \theta & -s_{1} \sin \theta+s_{2} \cos \theta\end{array}\right]\right)$

Denote $\sin \theta, \cos \theta, \tan \theta$ and $e^{-\mathbf{i} \pi / 4}$ by $s, c, t$ and $\epsilon$, respectively, we have

$$
\begin{aligned}
0= & \left(s_{1} c+s_{2} s\right)\left(-s_{1} s+s_{2} c\right)-\epsilon\left(-s_{5} s+s_{6} c\right)\left(s_{3} c+s_{4} s\right) \\
= & c^{2}\left[-\left(s_{1} s_{2}-\epsilon s_{4} s_{5}\right) t^{2}\right. \\
& \left.+\left(-s_{1}^{2}+s_{2}^{2}-\epsilon\left(-s_{3} s_{5}+s_{4} s_{6}\right)\right) t+s_{1} s_{2}-\epsilon s_{3} s_{6}\right]
\end{aligned}
$$

Since $t=\tan \theta \notin \mathbb{Q}$, where $\mathbb{Q}$ is the rational number field, and the minimal polynomial of $t$ over $\mathbb{Z}[\mathbf{i}]$ is $f(x)=x^{2}+x-1$, we have that $s_{i}, i=1,2, \cdots, 6$, satisfy $-\left(s_{1} s_{2}-\epsilon s_{4} s_{5}\right)=$ $-s_{1}^{2}+s_{2}^{2}-\epsilon\left(-s_{3} s_{5}+s_{4} s_{6}\right)=-\left(s_{1} s_{2}-\epsilon s_{3} s_{6}\right) \neq 0$ or $-\left(s_{1} s_{2}-\epsilon s_{4} s_{5}\right)=-s_{1}^{2}+s_{2}^{2}-\epsilon\left(-s_{3} s_{5}+s_{4} s_{6}\right)=s_{1} s_{2}-$ $\epsilon s_{3} s_{6}=0$.

Case 1. $-\left(s_{1} s_{2}-\epsilon s_{4} s_{5}\right)=-s_{1}^{2}+s_{2}^{2}-\epsilon\left(-s_{3} s_{5}+s_{4} s_{6}\right)=$ $s_{1} s_{2}-\epsilon s_{3} s_{6}=0$.

Since $\epsilon=e^{-\mathbf{i} \pi / 4} \notin \mathbb{Q}(\mathbf{i})$, where $\mathbb{Q}(\mathbf{i})$ is the number field generated by the rational number field $\mathbb{Q}$ and $\mathbf{i}$, equation $a+$ $\epsilon b=0$ holds for $a, b \in \mathbb{Z}[\mathbf{i}]$ if and only if $a=b=0$. Thus, in this case, we have $s_{1} s_{2}=s_{4} s_{5}=0,-s_{1}^{2}+s_{2}^{2}=$ $-s_{3} s_{5}+s_{4} s_{6}=0$ and $s_{1} s_{2}=s_{3} s_{6}=0$, which implies $s_{1}=s_{2}=s_{3}=s_{4}=0$ or $s_{1}=s_{2}=s_{5}=s_{6}=0$.

Case 2. $-\left(s_{1} s_{2}-\epsilon s_{4} s_{5}\right)=-s_{1}^{2}+s_{2}^{2}-\epsilon\left(-s_{3} s_{5}+s_{4} s_{6}\right)=$ $-\left(s_{1} s_{2}-\epsilon s_{3} s_{6}\right) \neq 0$.

From the equation $-\left(s_{1} s_{2}-\epsilon s_{4} s_{5}\right)=-s_{1}^{2}+s_{2}^{2}-\epsilon\left(-s_{3} s_{5}+\right.$ $s_{4} s_{6}$ ), we have

$$
s_{2}^{2}+s_{1} s_{2}-s_{1}^{2}+\epsilon\left(s_{3} s_{5}-s_{4} s_{5}-s_{4} s_{6}\right)=0 .
$$

Since $\epsilon \notin \mathbb{Q}(\mathbf{i})$, the above equation holds if and only if $s_{2}^{2}+$ $s_{1} s_{2}-s_{1}^{2}=0$ and $s_{3} s_{5}-s_{4} s_{5}-s_{4} s_{6}=0$. If $s_{1} \neq 0$, then $\left(\frac{s_{2}}{s_{1}}\right)^{2}+\frac{s_{2}}{s_{1}}-1=0$. We know that the roots of polynomial $x^{2}+x-1=0$ are $t$ and $-1-t \notin \mathbb{Q}(\mathbf{i})$. Thus, the equation $\left(\frac{s_{2}}{s_{1}}\right)^{2}+\frac{s_{2}}{s_{1}}-1=0$ does not hold for any $s_{1}, s_{2} \in \mathbb{Z}[\mathbf{i}]$ with $s_{1} \neq 0$. Otherwise, $s_{1}=0$, then $s_{2}$ should also be 0 .

From $-\left(s_{1} s_{2}-\epsilon s_{4} s_{5}\right)=-\left(s_{1} s_{2}-\epsilon s_{3} s_{6}\right)$, we have $s_{4} s_{5}=$ $s_{3} s_{6}$. Suppose $s_{4} s_{5}=s_{3} s_{6} \neq 0$, let $\frac{s_{3}}{s_{4}}=\frac{s_{5}}{s_{6}}=k \in \mathbb{Q}(\mathbf{i})$, and substituting $s_{3}$ and $s_{5}$ by $k s_{4}$ and $k s_{6}$ in the equation $s_{3} s_{5}-s_{4} s_{5}-s_{4} s_{6}=0$, we have

$$
s_{4} s_{6}\left(k^{2}-k-1\right)=0 .
$$

Similarly, $k^{2}-k-1 \neq 0$ for $k \in \mathbb{Q}(\mathbf{i})$, so one of $s_{4}$ and $s_{6}$ equals to 0 , which contradicts to the assumption. Thus, $s_{4} s_{5}=s_{3} s_{6}=0$. Together with $s_{3} s_{5}-s_{4} s_{5}-s_{4} s_{6}=0$, we can easily prove that $s_{3}=s_{4}=0$ or $s_{5}=s_{6}=0$. Similar to Case 1 , now we have $s_{1}=s_{2}=s_{3}=s_{4}=0$ or $s_{1}=s_{2}=s_{5}=s_{6}=0$.

For another $2 \times 2$ minor of matrix $\mathbf{X}$,

$$
\operatorname{det}\left(\left[\begin{array}{cc}
s_{3} \cos \theta+s_{4} \sin \theta & -s_{1} \sin \theta+s_{2} \cos \theta \\
e^{-\mathbf{i} \pi / 4}\left(s_{5} \cos \theta+s_{6} \sin \theta\right) & -s_{3} \sin \theta+s_{4} \cos \theta
\end{array}\right]\right) \text {. }
$$

Similar to the proof above, we have $s_{3}=s_{4}=s_{1}=s_{2}=0$ or $s_{3}=s_{4}=s_{5}=s_{6}=0$.

Hence, if all the minors of matrix $\mathbf{X}$ are zeros, we have $s_{1}=s_{2}=s_{3}=s_{4}=s_{5}=s_{6}=0$, which contradicts to that at least one of $s_{i}$ is not 0 . The matrix $\mathbf{X}$, consequently, has full rank.

For a general case, consider an $M$-layer code (19), in the next page, for $n_{t}$ transmit antennas with $P$ time slots, where $P-n_{t}+1 \leq M \leq P$, where $\rho_{1}, \cdots, \rho_{M}$ are $M$ fixed complex numbers, the $i$-th descending diagonal from left to right, denoted by $\mathbf{X}_{i}=\left[X_{i, 1}, X_{i, 2}, \cdots, X_{i, n_{t}}\right]^{T}$ is given by

$$
\mathbf{X}_{i}=\Theta \mathbf{s}_{i},
$$

where the $n_{t} \times 1$ information symbol vector $\mathbf{s}_{i}$ is

$$
\mathbf{s}_{i}=\left[s_{(i-1) n_{t}+1}, s_{(i-1) n_{t}+2}, \cdots, s_{i n_{t}}\right]^{T},
$$

$i=1,2, \cdots, M$, and $\Theta$ is a chosen constellation rotation matrix [34]. The symbol rate for this code is $\frac{M n_{t}}{P}$.

We can choose the rotation matrix $\Theta$ from [34], Table I. For a pair of integers $(l, m)$ and $K=l m, n_{t}=\frac{\phi(K)}{\phi(l)}$, where $\phi$ is the Euler totient function, a vaild rotation matrix is given by

$$
\Theta=\left[\begin{array}{cccc}
\zeta_{K} & \zeta_{K}^{2} & \cdots & \zeta_{K}^{n_{t}} \\
\zeta_{K}^{1+m_{2} l} & \zeta_{K}^{2\left(1+m_{2} l\right)} & \cdots & \zeta_{K}^{n_{t}\left(1+m_{2} l\right)} \\
\vdots & \vdots & \vdots & \vdots \\
\zeta_{K}^{1+m_{n_{t}} l} & \zeta_{K}^{2\left(1+m_{n_{t}} l\right)} & \cdots & \zeta_{K}^{n_{t}\left(1+m_{n_{t}} l\right)}
\end{array}\right]
$$

where $\zeta_{K}=\exp (2 \pi \mathbf{i} / K)$ and $m_{2}, m_{3}, \cdots, m_{n_{t}}$ are distinct integers such that $1+m_{i} l$ and $K$ are co-prime for any $2 \leq$ $i \leq n_{t}$. A signal constellation for this code can be $\Lambda_{\zeta_{l}}$ (or a subset $\mathcal{A}$ of $\Lambda_{\zeta_{l}}$ ), a subset of $\mathbb{Z}\left[\zeta_{l}\right]$ that is the number ring generated by the integer ring $\mathbb{Z}$ and $\zeta_{l}$, i.e., $\Lambda_{\zeta_{l}} \subset \mathbb{Z}\left[\zeta_{l}\right] . \Lambda_{\zeta_{l}}$ can also be thought of as the 2-dimensional real lattice with the generating matrix

$$
\left[\begin{array}{ll}
1 & \cos \left(\frac{2 \pi}{l}\right) \\
0 & \sin \left(\frac{2 \pi}{l}\right)
\end{array}\right]
$$

defined in [34]. For example, when $l=4$, the constellation is located on the square lattice, i.e., a QAM constellation. When 


\begin{tabular}{|c|c|c|c|c|c|c|c|}
\hline & & & $\mathbf{X}=$ & & & & \\
\hline$\rho_{1} X_{1,1}$ & 0 & $\cdots$ & 0 & $\rho_{M} X_{M, P-M+2}$ & $\cdots$ & $\rho_{P-n_{t}+2} X_{P-n_{t}+2, n_{t}}$ & \\
\hline$\rho_{2} X_{2,1}$ & $\rho_{1} X_{1,2}$ & $\ddots$ & $\vdots$ & 0 & $\ddots$ & $\vdots$ & \\
\hline$\vdots$ & $\rho_{2} X_{2,2}$ & $\ddots$ & 0 & $\vdots$ & $\ddots$ & $\rho_{M} X_{M, n_{t}}$ & \\
\hline$\rho_{M-1} X_{M-1,1}$ & & $\ddots$ & $\rho_{1} X_{1, P-M+1}$ & 0 & $\ddots$ & $\cdot$ & (19) \\
\hline$\rho_{M} X_{M, 1}$ & $\rho_{M-1} X_{M-1,2}$ & $\ddots$ & $\rho_{2} X_{2, P-M+1}$ & $\rho_{1} X_{1, P-M+2}$ & $\ddots$ & 0 & \\
\hline 0 & $\rho_{M} X_{M, 2}$ & $\ddots$ & $\vdots$ & $\rho_{2} X_{2, P-M+2}$ & $\ddots$ & $\rho_{1} X_{1, n_{t}}$ & \\
\hline$\vdots$ & $\vdots$ & $\ddots$ & $\rho_{M-1} X_{M-1, P-M+1}$ & $\vdots$ & $\ddots$ & $\vdots$ & \\
\hline 0 & 0 & $\cdots$ & $\rho_{M} X_{M, P-M+1}$ & $\rho_{M-1} X_{M-1, P-M+2}$ & $\cdots$ & $\rho_{P-n_{t}+1} X_{P-n_{t}+1, n_{t}}$ & \\
\hline
\end{tabular}

$l=3$, the signal constellation is located on the equal literal triangular lattice.

To decode this code with the conditional PIC-SIC group decoding, we first define the group ordering scheme $\mathcal{I}=$ $\left\{\mathcal{I}_{1}, \mathcal{I}_{2}, \cdots, \mathcal{I}_{P-n_{t}+2}\right\}$, where

$\mathcal{I}_{i}=\left\{(i-1) n_{t}+1,(i-1) n_{t}+2, \cdots, i n_{t}\right\}, i=1,2, \cdots, P-n_{t}+1$

and $\mathcal{I}_{P-n_{t}+2}=\left\{\left(P-n_{t}+1\right) n_{t}+1,\left(P-n_{t}+1\right) n_{t}+\right.$ $\left.2, \cdots, M n_{t}\right\}$.

When $P=n_{t}$, the matrix $\mathbf{X}$ in (19) is a square matrix, and this code is exactly the multilayer cyclotomic code proposed in [33]. In this case, we split the information symbols into two groups as above: $\mathcal{I}=\left\{\mathcal{I}_{1}, \mathcal{I}_{2}\right\}$, where $\mathcal{I}_{1}=\left\{1,2, \cdots, n_{t}\right\}$ and $\mathcal{I}_{2}=\left\{n_{t}+1, n_{t}+2, \cdots, M n_{t}\right\}$. When we use the conditional PIC-SIC group decoding with this grouping scheme, the decoding is equivalent to the ML decoding.

When $P>n_{t}$, with $M$ layers, the symbol rate of this code is $\frac{M n_{t}}{P}$. With the grouping scheme $\mathcal{I}=$ $\left\{\mathcal{I}_{1}, \mathcal{I}_{2}, \cdots, \mathcal{I}_{P-n_{t}+2}\right\}$ as above, we use the conditional PICSIC group decoding to decode the information symbols as follows: first, for every possible trail of the symbols in the last group $\mathbf{s}_{\mathcal{I}_{P-n_{t}+2}}$, estimate the information symbols in other symbol groups $\mathbf{s}_{\mathcal{I}_{1}}, \mathbf{s}_{\mathcal{I}_{2}}, \cdots, \mathbf{s}_{\mathcal{I}_{P-n_{t}+1}}$ with the PIC-SIC group decoding; then choose the best $\mathbf{s}_{\mathcal{I}_{P-n_{t}+2}}$ to minimize the ML metric from all results in the first step. The decoding complexities of the code $\mathbf{X}$ in (19) in the first step and second step are $O\left(|\mathcal{A}|^{n_{t}}\right)$ and $O\left(|\mathcal{A}|^{n_{t}\left(M+n_{t}-P-1\right)}\right)$, respectively. Thus, the total decoding complexity is $O\left(|\mathcal{A}|^{n_{t}\left(M+n_{t}-P\right)}\right)$. For the multilayer cyclotomic space time code proposed in [33] with the ML decoding, if the decoding complexity is the same as $\mathbf{X}$ in (19), i.e., $O\left(|\mathcal{A}|^{n_{t}\left(M+n_{t}-P\right)}\right)$, the multilayer cyclotomic space time code should have $M+n_{t}-P$ layers with $n_{t}$ transmit antennas and $n_{t}$ time slots. So the corresponding symbol rate is $M+n_{t}-P$. It is not hard to see that this symbol rate is less than the symbol rate of the proposed code in (19), since

$$
\begin{aligned}
\left(M+n_{t}-P\right)-\frac{M n_{t}}{P} & =\frac{1}{P}\left(M P+n_{t} P-P^{2}-M n_{t}\right) \\
& =\frac{1}{P}\left(P-n_{t}\right)(M-P) \leq 0
\end{aligned}
$$

where the last inequality is obtained from $P>n_{t}$ and $M \leq$ $P$. The above less than sign $\leq$ holds strictly, i.e., $<$, when
$P>M$.

Comparing with the code proposed in [28], when $M=$ $P-n_{t}+1$, the last group $\mathcal{I}_{P-n_{t}+2}$ does not appear and the proposed code is the same that in [28]. For $M>P-n_{t}+1$, the symbol rate of the proposed code, $\frac{M n_{t}}{P}$, is always greater than the symbol rate of the code in [28] with the same numbers of transmit antennas and time slots, which is $\frac{n_{t}\left(P-n_{t}+1\right)}{P}$. The decoding complexity of the proposed code with the conditional PIC-SIC group decoding is, however, higher than that of the code in [28] with the PIC-SIC group decoding.

The following property guarantees that the code $\mathbf{X}$ above achieves the full diversity with the conditional PIC-SIC group decoding.

Property 4.2: For the STBC $\mathbf{X}$ in (19), if the received signal is decoded using the conditional PIC-SIC group decoding with the group ordering scheme $\mathcal{I}=\left\{\mathcal{I}_{1}, \mathcal{I}_{2}, \cdots, \mathcal{I}_{P-n_{t}+2}\right\}$, then the code $\mathbf{X}$ achieves the full diversity, when $\rho_{i}=\rho_{0}^{i-1}, i=$ $1, \cdots, M$, satisfy one of the following conditions:

1) $\rho_{0}=\zeta_{n}$ with $n=n_{0} K$ and $n_{0}=p_{1}^{r_{1}} p_{2}^{r_{2}} \cdots p_{u}^{r_{u}}, n_{0} \geq$ $n_{t}(M-1)+1$, where $p_{1}, \cdots, p_{u}$ are some prime factors of $K$;

2) $\rho_{0}=e^{j \lambda}$ for an algebraic number $\lambda \neq 0$, i.e., $\rho_{0}$ is transcendental;

3) $\rho_{0}=\sqrt{\beta}^{1 / n_{t}} \zeta_{n^{\prime}}$ with a proper integer $\beta$ and $n^{\prime} \leq n$ with the same $n$ as in 1).

Proof: Suppose that only one receive antenna is used and the channel matrix $\mathbf{H}=\left[h_{1}, h_{2}, \cdots, h_{n_{t}}\right]^{T}$. For multiple receive antennas, the proof is similar. Let the rotation matrix $\Theta=\left[\Theta_{1}^{T}, \Theta_{2}^{T}, \cdots, \Theta_{n_{t}}^{T}\right]^{T}$, where $\Theta_{i}=$ $\left[\zeta_{K}^{1+m_{i} l}, \zeta_{K}^{2\left(1+m_{i} l\right)}, \cdots, \zeta_{K}^{n_{t}\left(1+m_{i} l\right)}\right]$ with $m_{1}=0$, i.e., $\Theta_{i}$ be the $i$ th row vector of $\Theta$; and $\mathbf{g}_{i}=h_{i} \Theta_{i}, i=1,2, \cdots, n_{t}$. The equivalent channel matrix is (22) in the next page, where for $i=1,2, \cdots, P-n_{t}+1$,

$$
\mathbf{G}_{\mathcal{I}_{i}}=\left[\begin{array}{c}
\mathbf{0}_{(i-1) \times n_{t}} \\
\rho_{i} \operatorname{diag}(H) \Theta \\
\mathbf{0}_{\left(P-n_{t}-i+1\right) \times n_{t}}
\end{array}\right],
$$

and $\mathbf{G}_{\mathcal{I}_{P-n_{t}+2}}$ can be expressed as (23) in the next page.

For a nonzero codeword, since the symmetry structure of the codeword, we can suppose $\mathbf{X}_{1}$ in (19)-(20) is nonzero. First, to prove the code $\mathbf{X}$ has full rank, we want to show that the determinant of the first $n_{t}$ rows of $\mathbf{X}$ does not equal to zero. It 


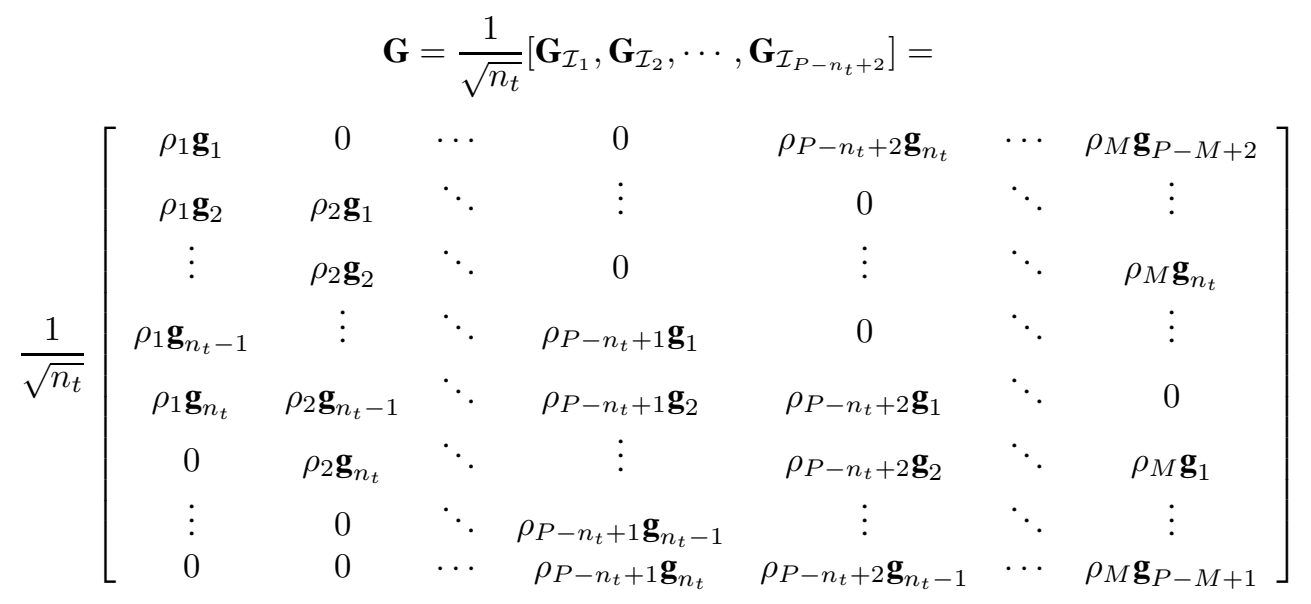

$$
\mathbf{G}_{\mathcal{I}_{P-n_{t}+2}}=\left[\begin{array}{cccc}
\rho_{P-n_{t}+2} \mathbf{g}_{n_{t}} & \rho_{P-n_{t}+3} \mathbf{g}_{n_{t}-1} & \cdots & \rho_{M} \mathbf{g}_{P-M+2} \\
0 & \rho_{P-n_{t}+3} \mathbf{g}_{n_{t}} & \ddots & \vdots \\
\vdots & 0 & \ddots & \rho_{M} \mathbf{g}_{n_{t}} \\
0 & \vdots & \ddots & \vdots \\
\rho_{P-n_{t}+2} \mathbf{g}_{1} & 0 & \ddots & 0 \\
\rho_{P-n_{t}+2} \mathbf{g}_{2} & \rho_{P-n_{t}+3} \mathbf{g}_{1} & \ddots & \rho_{M} \mathbf{g}_{1} \\
\vdots & \vdots & \ddots & \vdots \\
\rho_{P-n_{t}+2} \mathbf{g}_{n_{t}-1} & \rho_{P-n_{t}+3} \mathbf{g}_{n_{t}-2} & \cdots & \rho_{M} \mathbf{g}_{P-M+1}
\end{array}\right] .
$$

is not hard to see that the determinant is a nonzero polynomial of $\rho_{0}$ of order no more than $n_{t}(M-1)$ with coefficients in $\mathbb{Q}\left(\zeta_{K}\right)$, where $\mathbb{Q}\left(\zeta_{K}\right)$ is the number field generated by the rational number field $\mathbb{Q}$ and $\zeta_{K}$. Thus, the full rank property is equivalent to stating that $\rho_{0}$ is not a root of such a polynomial. The proof of this property is the same as the proof of Theorem 2 in [33].

Second, suppose the channel coefficients $h_{1}=h_{2}=\cdots=$ $h_{j-1}=0$ and $h_{j} \neq 0$. By Theorem 2 in [34], any nonzero linear combination over $\Delta \Lambda_{\zeta_{l}}$ of the entries of $\Theta_{j}$ is not zero, i.e.,

$$
a_{1} \zeta_{K}^{1+m_{j} l}+a_{2} \zeta_{K}^{2\left(1+m_{j} l\right)}+\cdots+a_{n_{t}} \zeta_{K}^{n_{t}\left(1+m_{j} l\right)} \neq 0,
$$

for $\left[a_{1}, a_{2}, \cdots, a_{n_{t}}\right] \neq[0,0, \cdots, 0]$ and $a_{1}, a_{2}, \cdots, a_{n_{t}} \in$ $\Delta \Lambda_{\zeta_{l}}$. Thus, any nonzero linear combination over $\Delta \Lambda_{\zeta_{l}}$ of the entries of the $(i+j-1)$-th row $\mathbf{G}_{\mathcal{I}_{i}}, \rho_{i} h_{j} \Theta_{j}$, is nonzero. At the same time, the entries in the same row of $\mathbf{G}_{\mathcal{I}_{k}}$ are either $\rho_{k} \mathbf{g}_{j+i-k}=\rho_{k} h_{j+i-k} \Theta_{j+i-k}$ with $h_{j+i-k}=0$, i.e., 0 , for $k=i+1, i+2, \cdots, j+i-1$ or 0 for $k=j+i, j+i+1, \cdots, P-n_{t}+1$, which implies that any nonzero linear combination over $\Delta \Lambda_{\zeta_{l}}$ of the vectors in $G_{\mathcal{I}_{i}}$ does not belong to the space linearly spanned by all the vectors in the group $\left[\boldsymbol{G}_{\mathcal{I}_{i+1}}, \cdots, \boldsymbol{G}_{\mathcal{I}_{P-n_{t}+1}}\right]$ over the complex field for any $\mathbf{H} \neq 0$.

Thus, this code can achieve the full diversity using the conditional PIC-SIC group decoding, since the two conditions in Theorem 3.2 are satisfied.

When $M=P-n_{t}+2$, the last group of information symbol indices is $\mathcal{I}_{M}=\left\{(M-1) n_{t}+1, \cdots, M n_{t}\right\}$ that has $n_{t}$ symbols from the last layer in code $\mathbf{X}$. In this case, we have the following simplified result.

Property 4.3: For the STBC $\mathbf{X}$ in (19), if the received signal is decoded using the conditional PIC-SIC group decoding with the group ordering scheme $\mathcal{I}=\left\{\mathcal{I}_{1}, \mathcal{I}_{2}, \cdots, \mathcal{I}_{P-n_{t}+2}\right\}$, then the code $\mathbf{X}$ achieves the full diversity, when $M=P-n_{t}+2$, $\rho_{i}=1, i=1,2, \cdots, M-1$, and $\rho_{M}=\zeta_{n}$ with $n=n_{0} K$ and $n_{0}=p_{1}^{r_{1}} p_{2}^{r_{2}} \cdots p_{u}^{r_{u}}$, where $p_{1}, \cdots, p_{u}$ are some prime factors of $K$, such that $n_{0} \geq n_{t}-M+3$ for $n_{t} \geq M$ or $n_{0} \geq 2$ for $n_{t}<M$.

Proof: Similar to the proof of Property 4.2, we can show that this code satisfies the second condition in Theorem 3.2. So we only need to prove that the codeword matrix,

$\mathbf{X}=\left[\begin{array}{ccccc}X_{1,1} & 0 & \cdots & 0 & \rho_{M} X_{M, n_{t}} \\ X_{2,1} & X_{1,2} & \ddots & \vdots & 0 \\ \vdots & X_{2,2} & \ddots & 0 & \vdots \\ X_{M-1,1} & \vdots & \ddots & X_{1, n_{t}-1} & 0 \\ \rho_{M} X_{M, 1} & X_{M-1,2} & \ddots & X_{2, n_{t}-1} & X_{1, n_{t}} \\ 0 & \rho_{M} X_{M, 2} & \ddots & \vdots & X_{2, n_{t}} \\ \vdots & \vdots & \ddots & X_{M-1, n_{t}-1} & \vdots \\ 0 & 0 & \cdots & \rho_{M} X_{M, n_{t}-1} & X_{M-1, n_{t}}\end{array}\right]$

has full rank with the information symbols $s_{i} \in \mathbb{Z}\left[\zeta_{l}\right]$, for $i=1,2, \cdots, M n_{t}$, where at least one of $s_{i}$ is not zero.

If $\mathbf{X}_{1}=\left[X_{1,1}, X_{1,2}, \cdots, X_{1, n_{t}}\right]^{T}=\mathbf{0}$, by the row permu- 
tation, we can express the code $\mathbf{X}$ in (24) as follows:

$$
\mathbf{X}=\left[\begin{array}{ccccc}
X_{2,1} & 0 & \cdots & 0 & 0 \\
\vdots & X_{2,2} & \ddots & \vdots & \vdots \\
X_{M-1,1} & \vdots & \ddots & 0 & 0 \\
\rho_{M} X_{M, 1} & X_{M-1,2} & \ddots & X_{2, n_{t}-1} & 0 \\
0 & \rho_{M} X_{M, 2} & \ddots & \vdots & X_{2, n_{t}} \\
\vdots & \vdots & \ddots & X_{M-1, n_{t}-1} & \vdots \\
0 & 0 & \ddots & \rho_{M} X_{M, n_{t}-1} & X_{M-1, n_{t}} \\
0 & 0 & \cdots & 0 & \rho_{M} X_{M, n_{t}}
\end{array}\right]
$$

Similarly to the code $C_{4,6,3}$ in [28], we can show that the code $\mathbf{X}$ in (25) has the full rank property.

Otherwise, when $\mathbf{X}_{1} \neq \mathbf{0}$, if $n_{t} \geq M$, consider the square matrix (26), in the next page, of the first $n_{t}$ rows of the code $\mathbf{X}$ in (24). The determinant of this square matrix is a nonzero polynomial of $\rho_{M}$ of the order no more than $n_{t}-M+2$ with the coefficients in $\mathbb{Q}\left(\zeta_{K}\right)$. By the definition of $n$, we have that $\mathbb{Q}\left(\zeta_{K}\right) \subset \mathbb{Q}\left(\zeta_{n}\right)$. The dimension of the vector space $\mathbb{Q}\left(\zeta_{n}\right)$ over the field $\mathbb{Q}\left(\zeta_{K}\right)$ is

$$
\left[\mathbb{Q}\left(\zeta_{n}\right): \mathbb{Q}\left(\zeta_{K}\right)\right]=\frac{\phi(n)}{\phi(K)}=n_{0} \geq n_{t}-M+3 .
$$

Thus, we have that the minimal polynomial of $\zeta_{n}$ over field $\mathbb{Q}\left(\zeta_{K}\right)$ is of the order $n_{0}$, which implies that the determinant of this square matrix does not equal to zero. Hence, the code $\mathbf{X}$ has the full rank property.

If $n_{t}<M$, the square matrix of the first $n_{t}$ rows of the code $\mathbf{X}$ in (24) can be expressed as

$$
\left[\begin{array}{ccccc}
X_{1,1} & 0 & \cdots & 0 & \rho_{M} X_{M, n_{t}} \\
X_{2,1} & X_{1,2} & \ddots & \vdots & 0 \\
\vdots & X_{2,2} & \ddots & 0 & \vdots \\
X_{n_{t}-1,1} & \vdots & \ddots & X_{1, n_{t}-1} & 0 \\
X_{n_{t}, 1} & X_{n_{t}-1,2} & \cdots & X_{2, n_{t}-1} & X_{1, n_{t}}
\end{array}\right] .
$$

The determinant of this square matrix is a nonzero polynomial of $\rho_{M}$ of the order at most 1 with the coefficients in $\mathbb{Q}\left(\zeta_{K}\right)$. Similarly, the square matrix has the full rank with $n_{0} \geq 2$.

Thus, we have proved that the code $\mathbf{X}$ in (24) has the full rank property. Therefore, this code can achieve the full diversity using the conditional PIC-SIC group decoding.

\section{Numerical Simulations}

In this section, we present some simulation results for the cases of (i) two transmit and three receive antennas, and (ii) four transmit and four receive antennas. The channel is assumed quasi-static Rayleigh flat fading.

For Case (i), we compare our proposed code (17) with the code (called Guo-Xia code for convenience) in [26]. In Fig.1, the bandwidth efficiencies are $8 \mathrm{bits} / \mathrm{sec} / \mathrm{Hz}$ for all the codes. For Guo-Xia code, we use the PIC group decoding. For our proposed code, we use the conditional PIC group decoding. From Fig.1, it is easily observed that the new code offers better performance than Guo-Xia code in [26], while its decoding complexity is higher. We also show the performances of the proposed code (17) with two different decoding methods: the conditional PIC group decoding with sphere decoding in the ML step marked by $\diamond$ and the conditional PIC group decoding with sphere decoding in both the ML step and the PIC group decoding step marked by + . These performances are similar to that of the conditional PIC group decoding.

For Case (ii), we compare our proposed code (19) with the parameters in Property 4.3 with $n_{0}=4$ and $K=16$ of symbol rate $8 / 3$, with the perfect code in [31] of symbol rate 4 , and with the code $C_{4,6,3}$ in [28] of symbol rate 2. In both Fig. 2 and Fig.3, the group ordering schemes of the conditional PIC-SIC group decoding are $\{\{1,2,3,4\},\{5,6,7,8\},\{9,10,11,12\},\{13,14,15,16\}\}$ for the proposed code and the perfect code and $\{\{1,2,3,4\},\{5,6,7,8\},\{9,10,11,12\}\}$ for the code $C_{4,6,3}$, respectively. In Fig.2, the code $C_{4,6,3}$ gives the best performance, mainly because it has a lower bandwidth efficiency than the proposed code. We can see that the perfect code cannot achieve the full diversity with the conditional PIC-SIC group decoding. Even with a higher bandwidth efficiency, the proposed code has a better performance than the perfect code with the same conditional PIC-SIC group decoding. We also show the performances of the proposed code using the conditional PIC group decoding with sphere decoding in the ML step marked by $\diamond$ and in both the ML decoding and the PIC group decoding steps marked by +. In Fig.3, the bandwidth efficiencies for all codes are 8 bits/s/Hz. The perfect code with the ML decoding has the best performance. However, without the full diversity, the perfect code with the conditional PIC-SIC group decoding performs worse than the proposed code at high SNRs. Comparing to the code $C_{4,6,3}$ with the ML decoding, the proposed code with the conditional PIC-SIC group decoding has a better performance. As a result, the proposed code will give a better performance than the code $C_{4,6,3}$ with the conditional PIC-SIC group decoding, while $C_{4,6,3}$ is proposed in [28] for the PIC-SIC group decoding that has a lower complexity than the conditional PIC-SIC group decoding proposed in this paper does.

\section{CONCLUSION}

In this paper, we proposed a conditional PIC group decoding whose complexity is between those of the PIC group decoding and the ML decoding. We then obtained a new STBC design criterion for full diversity achieving STBC with the conditional PIC group decoding, which is also between those of the PIC group decoding and the ML decoding. Finally, we proposed some new STBC designs that satisfy the new criterion and therefore achieve full diversity with the conditional PIC (or PIC-SIC) group decoding and in the meantime, have higher symbol rates than those designed for the PIC (or PIC-SIC) group decoding.

\section{ACKNOWLEDGMENT}

The authors would like to thank one of the reviewers to suggest them to consider combining sphere decoding with the 


$$
\left[\begin{array}{ccccccc}
X_{1,1} & 0 & \cdots & 0 & \cdots & 0 & \rho_{M} X_{M, n_{t}} \\
X_{2,1} & X_{1,2} & \ddots & \vdots & \vdots & \vdots & 0 \\
\vdots & X_{2,2} & \ddots & 0 & \ddots & \vdots & \vdots \\
X_{M-1,1} & \vdots & \ddots & X_{1, n_{t}-M+1} & \ddots & \vdots & \vdots \\
\rho_{M} X_{M, 1} & X_{M-1,2} & \ddots & X_{2, n_{t}-M+1} & \ddots & \vdots & \vdots \\
0 & \rho_{M} X_{M, 2} & \ddots & \vdots & \ddots & 0 & \vdots \\
\vdots & \vdots & \ddots & X_{M-1, n_{t}-M+1} & \ddots & X_{1, n_{t}-1} & 0 \\
0 & 0 & \cdots & \rho_{M} X_{M, n_{t}-M+1} & \cdots & X_{2, n_{t}-1} & X_{1, n_{t}}
\end{array}\right]
$$

conditional PIC group decoding.

\section{REFERENCES}

[1] S. M. Alamouti, "A simple transmit diversity technique for wireless communication," IEEE J. Sel. Areas Commun., vol. 16, pp. 1451-1458, Oct. 1998.

[2] V. Tarokh, H. Jafarkhani, and A. R. Calderbank, "Space-time block codes from orthogonal designs," IEEE Trans. Inform. Theory, vol. 45, no. 5, pp. 1456-1467, Jul. 1999.

[3] G. Ganesan and P. Stoica, "Space-time block codes: a maximum SNR approach," IEEE Trans. Inform. Theory, vol. 47, no. 4, pp. 1650-1656, May 2001.

[4] H. Wang and X.-G. Xia, "Upper bounds of rates of complex orthogonal space-time block codes," IEEE Trans. Inform. Theory, vol. 49, no. 10 , pp. 2788-2796, Oct. 2003.

[5] X.-B. Liang, "Orthogonal designs with maximal rates," IEEE Trans. Inform. Theory, vol. 49, no. 10, pp. 2468-2503, Oct. 2003.

[6] W. Su, X.-G. Xia, and K. J. R. Liu, "A systematic design of high-rate complex orthogonal space-time block codes," IEEE Commun. Lett., vol. 8, no. 6, pp. 380-382, Jun. 2004.

[7] K. Lu, S. Fu, and X.-G. Xia, "Closed-form designs of complex orthogonal space-time block codes of rate $(2 k+1) / 2 k$ for $2 k-1$ or $2 k$ transmit antennas," IEEE Trans. Inform. Theory, vol. 51, no. 10, pp. 4340-4347, Oct. 2005.

[8] H. Jafarkhani, "A quasi-orthogonal space-time block codes," IEEE Trans. Commun., vol. 49, pp. 1-4, Jan. 2001.

[9] O. Tirkkonen, "Optimizing space-time block codes by constellation rotations," Proc. Finnish Wireless Commun. Workshop FWCW'01, Finland, pp.59-60, Oct. 2001.

[10] O. Tirkkonen, A. Boariu, and A. Hottinen, "Minimal non-orthogonality rate 1 space-time block code for 3-Tx antennas," in Proc. IEEE 6th Int Symp. on Spread-Spectrum Tech. and Appl., pp. 429-432, Sept. 2000.

[11] N. Sharma and C. B. Papadias, "Improved quasi-orthogonal codes through constellation rotation," IEEE Trans. Commun. vol. 51, No. 3 , pp. $332-335,2003$.

[12] W. Su and X.-G. Xia, "Signal constellations for quasi-orthogonal spacetime block codes with full diversity," IEEE Trans. Inform. Theory, vol. 50, pp. 2331 - 2347, Oct. 2004.

[13] Z. A. Khan and B. S. Rajan, "Single-symbol maximum-likelihood decodable linear STBCs," IEEE Trans. Inform. Theory, vol. 52, no. 5, pp. 2062-2091, May 2006.

[14] C. Yuen, Y. L. Guan, and T. T. Tjhung, "Quasi-orthogonal STBC with minimum decoding decoding complexity," IEEE Trans. Wireless Commun., vol. 4, pp. 2089-2094, Sept. 2005.

[15] D. N. Dao, C. Yuen, C. Tellambura, Y. L. Guan, and T. T. Tjhung, "Four-group decodable space-time block codes," IEEE Trans. Signal Processing, vol. 5, pp. 345 - 355, Jan. 2008.

[16] S. Karmakar and B. S. Rajan, "Multigroup decodable STBCs from Clifford algebra," IEEE Trans. Inform. Theory, vol. 55, pp. 223 - 231, Jan. 2009.

[17] S. Sezginer and H. Sari, "Full-rate full-diversity $2 \times 2$ space-time codes of reduced decoder complexity," IEEE Commun. Lett., vol. 11, no. 12 pp. 973-975, Dec. 2007.
[18] A. Ismail, H. Sari, J. Fiorina, and M. O. Damen, "A rate-3/2 fulldiversity $4 \times 4$ space-time code with fast maximum-likelihood decoding," Proc. of the 20th Commemorative Personal, Indoor and Mobile Radio Communications Symposium (PIMRC'09), Tokyo, Japan, Sept. 13-16, 2009.

[19] S. Sirianunpiboon, Y. Wu, A. R. Calderbank, and S. Howard, "Fast optimal decoding of multiplexed orthogonal designs by conditional optimization," IEEE Trans. Inform. Theory, vol. 56, No. 3, pp. 11061113, Mar. 2010.

[20] K. Bollapalli, Y. Wu, K. Gulati, S. Khatri, and A. R. Calderbank, "Highly parallel decoding of space-time codes on graphics processing units," the 47th Annual Allerton Conf. Communication, Control, and Computing, pp. 1262-1269, Allerton House, IL, USA, Sept. 30 - Oct. 2, 2009.

[21] J. Liu, J.-K. Zhang, and K. M. Wong, "On the design of minimum BER linear space-time block codes for MIMO systems equipped with MMSE receivers," IEEE Trans. Signal Processing, vol. 54, pp. 3147-3158, Aug. 2006.

[22] J. Liu, J.-K. Zhang, and K. M. Wong, "Full-diversity codes with MISO systems equipped with linear or ML detectors," IEEE Trans. Inform Theory, vol. 54, No. 10, pp. 4511-4527, Oct. 2008.

[23] Y. Shang and X.-G. Xia, "Space-time block codes achieving full diversity with linear receivers," IEEE Trans. Inform. Theory, vol. 54, No. 10 , pp. $4528-4547$, Oct. 2008

[24] W. Zhang and J. Yuan, "A simple design of space-time block codes achieving full diversity with linear receivers," in Proc. IEEE ICASSP, Taipei, Apr. 20-24, 2009.

[25] H.-M. Wang, X.-G. Xia, Q. Yin, and B. Li, "A family of space-time block codes achieving full diversity with linear receivers," IEEE Trans. Commun., vol.57, pp.3607-3617, Dec. 2009.

[26] X. Guo and X.-G. Xia, "On full diversity space-time block codes with partial interference cancellation group decoding," IEEE Trans. Inform. Theory, vol.55, pp.4366-4385, Oct. 2009.

[27] X. Guo and X.-G. Xia, "Correction to 'On full diversity space-time block codes with partial iinterference cancellation group decoding,," IEEE Trans. Inform. Theory, vol.56, pp.3635-3636, Jul. 2010.

[28] W. Zhang and X.-G. Xia, "A systematic design of space-time block codes achieving full-diversity with partial interference cancelation group decoding," Proc. Globecom 2009, Hawaii, Nov. 30-Dec. 4, 2009.

[29] Kiran T. and B. S. Rajan, "STBC-scheme with nonvanishing determinant for certain number of transmit antennas," IEEE Trans. Inform. Theory, vol. 51, no. 8, pp. 2984-2992, Aug. 2005.

[30] P. Elia, K. R. Kumar, S. A. Pawar, P. V. Kumar, and H.-F. Lu, "Explicit space-time codes achieving the diversity-multiplexing gain tradeoff," IEEE Trans. Inform. Theory, vol. 52, no. 9, pp. 3869-3884, Sept. 2006.

[31] F. Oggier, G. Rekaya, J.-C. Belfiore, and E. Viterbo, "Perfect space time block codes," IEEE Trans. Inform. Theory, vol. 52, no. 9, pp. 3885-3902, Sept. 2006.

[32] P. Elia, B. A. Sethuraman, and P. V. Kumar, "Perfect space-time codes for any number of antennas," IEEE Trans. Inform. Theory, vol. 53, no. 11, pp. 3853-3868, Nov. 2007.

[33] G. Wang and X.-G. Xia, "On optimal multilayer cyclotomic space-time code designs," IEEE Trans. Inform. Theory, vol.51, pp.1102-1135, Mar. 2005.

[34] G. Wang, H. Liao, H. Wang, and X.-G. Xia, "Systematic and optimal cyclotomic lattices and diagonal space-time block code designs," IEEE Trans. Inform. Theory, vol. 50, no. 12, Dec. 2004

[35] B. Hassibi and B. M. Hochwald, "High-rate codes that are linear in space 
and time," IEEE Trans. Inform. Theory, vol. 48, no. 7, pp. 1804-1824, Jul. 2002.

[36] J. R. W. Heath and A. J. Paulraj, "Linear dispersion codes for MIMO systems based on frame theory," IEEE Trans. Signal Process., vol. 50, no. 10, pp. 2429-2441, Oct. 2002.

[37] E. Viterbo and E. Biglieri, "A universal decoding algorithm for lattice codes," Proc. Quatorzieme Colloque GRETSI, pp. 611-614, Sept. 1993.

[38] E. Viterbo and J. Boutros, "A universal lattice decoder for fading channels," IEEE Trans. Inform. Theory, vol. 45, no. 5, pp. 1639-1642, Jul. 1999.

[39] M. O. Damen, K. Abed-Meraim, and J. C. Belfiore, "Generalized sphere decoder for asymmetrical space-time communication architecture," IEE Electron. Lett., vol. 36, pp. 166, Jan. 2000.

[40] M. O. Damen, A. Chkeif, and J. C. Belfiore, "Lattice code decoder for space-time codes," IEEE Commun. Lett., vol. 4, no. 5, pp. 161-163, May 2000.

[41] B. Hassibi and H. Vikalo, "On the sphere-decoding algorithm I. expected complexity," IEEE Trans. Signal Processing, vol. 53, no. 8, pp. 28062818, Aug. 2005.

[42] B. Hassibi and H. Vikalo, "On the sphere-decoding algorithm II. generalizations, second-order statistics, and applications to communications," IEEE Trans. Signal Processing, vol. 53, no. 8, pp. 2819-2834, Aug. 2005.

[43] W. H. Mow, "Universal lattice decoding: principle and recent advances," Wireless Comm. Mobile Comput., vol. 3, pp. 553-569, Aug. 2003. 


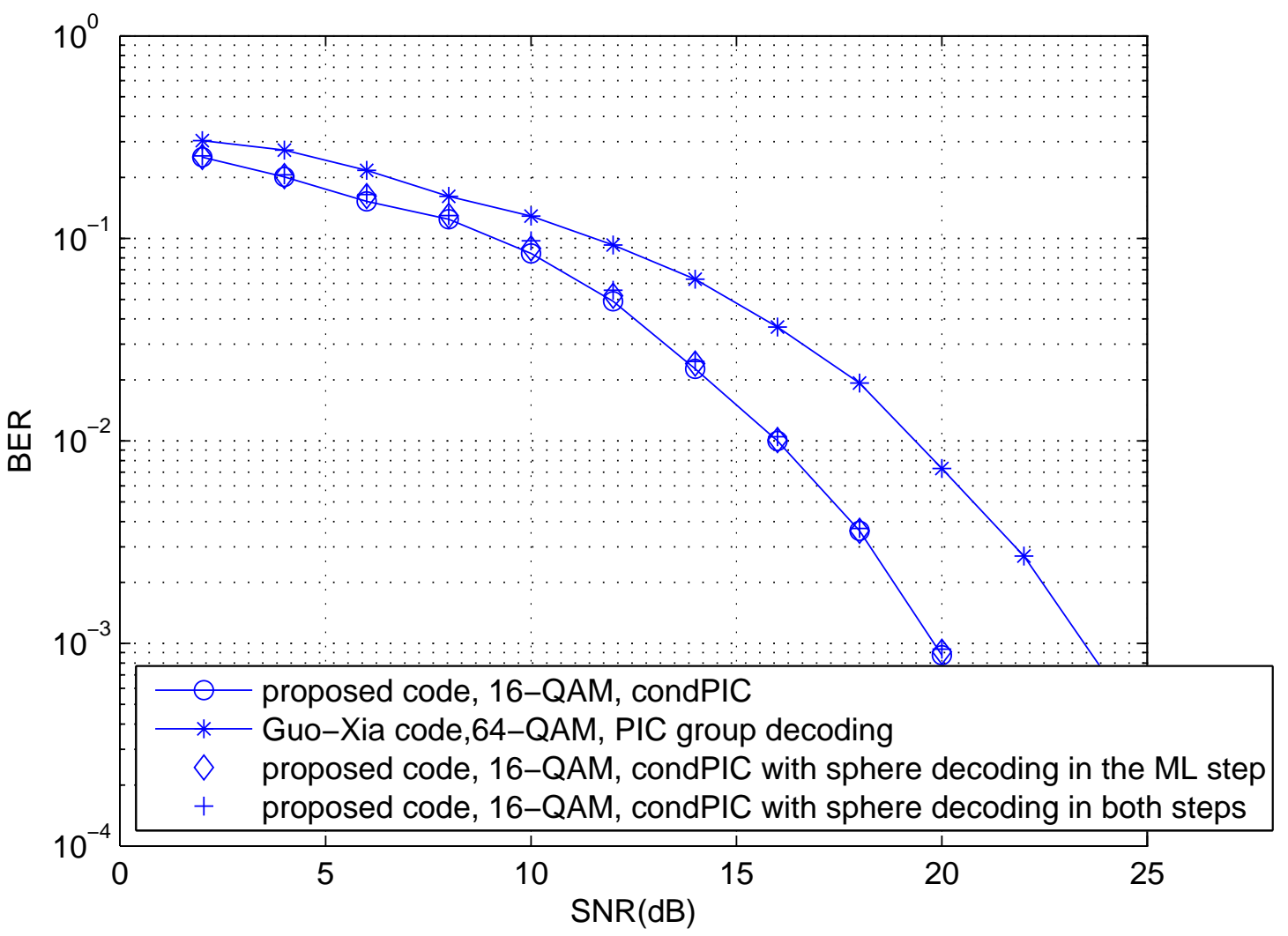

Fig. 1. Performance comparison of the new code and Guo-Xia code with bandwidth efficiency 8 bits/sec/Hz, two transmit and three receive antennas 


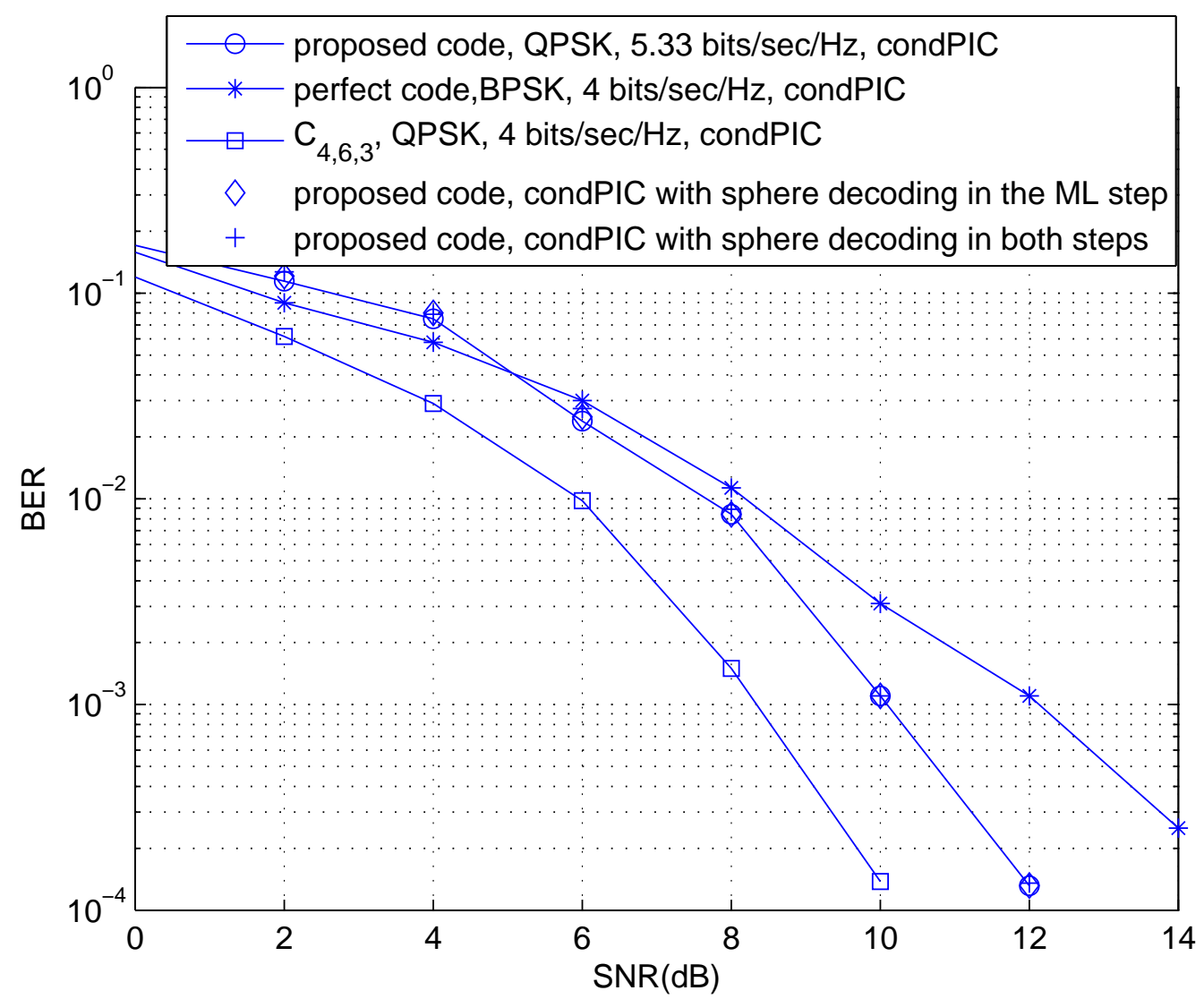

Fig. 2. Performance comparison of the proposed code (19), the perfect code, and the code $C_{4,6,3}$ using the conditional PIC-SIC group decoding with four transmit and four receive antennas 


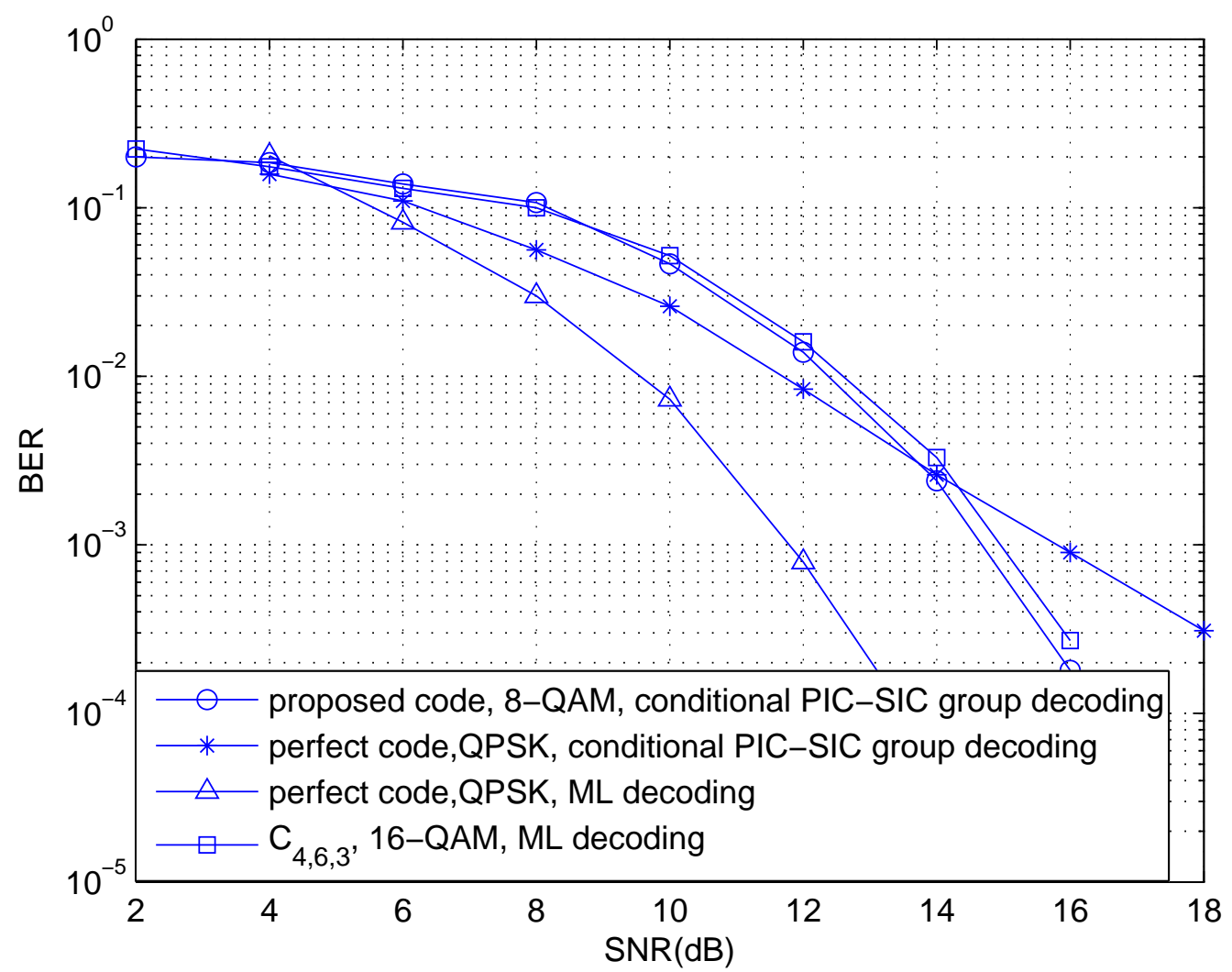

Fig. 3. Performance comparison of the proposed code (19), the perfect code, and the code $C_{4,6,3}$ with bandwidth efficiency $8 \mathrm{bits} / \mathrm{sec} / \mathrm{Hz}$, four transmit and four receive antennas 
Tianyi Xu received his B.S. degree in mathematics from University of Science and Technology of China, Hefei, China, and the M.S. degree in mathematics from Nankai University, Tianjin, China, in 2004 and 2007, respectively.

$\mathrm{He}$ is currently pursuing his Ph.D. degree at the University of Delaware, Newark, DE, USA. His current research interests are space-time coding for MIMO and distributed systems.

Xiang-Gen Xia (M'97, S'00, F'09) received his B.S. degree in mathematics from Nanjing Normal University, Nanjing, China, and his M.S. degree in mathematics from Nankai University, Tianjin, China, and his Ph.D. degree in Electrical Engineering from the University of Southern California, Los Angeles, in 1983, 1986, and 1992, respectively.

He was a Senior/Research Staff Member at Hughes Research Laboratories, Malibu, California, during 1995-1996. In September 1996, he joined the Department of Electrical and Computer Engineering, University of Delaware, Newark, Delaware, where he is the Charles Black Evans Professor. He was a Visiting Professor at the Chinese University of Hong Kong during 2002-2003, where he is an Adjunct Professor. Before 1995, he held visiting positions in a few institutions. His current research interests include space-time coding, MIMO and OFDM systems, digital signal processing, and SAR and ISAR imaging. Dr. Xia has over 200 refereed journal articles published and accepted, and 7 U.S. patents awarded and is the author of the book Modulated Coding for Intersymbol Interference Channels (New York, Marcel Dekker, 2000).

Dr. Xia received the National Science Foundation (NSF) Faculty Early Career Development (CAREER) Program Award in 1997, the Office of Naval Research (ONR) Young Investigator Award in 1998, and the Outstanding Overseas Young Investigator Award from the National Nature Science Foundation of China in 2001. He also received the Outstanding Junior Faculty Award of the Engineering School of the University of Delaware in 2001. $\mathrm{He}$ is currently an Associate Editor of the IEEE Transactions on Wireless Communications, IEEE Trasactions on Signal Processing, Signal Processing (EURASIP), and the Journal of Communications and Networks (JCN). He was a guest editor of Space-Time Coding and Its Applications in the EURASIP Journal of Applied Signal Processing in 2002. He served as an Associate Editor of the IEEE Transactions on Signal Processing during 1996 to 2003, the IEEE Transactions on Mobile Computing during 2001 to 2004, IEEE Transactions on Vehicular Technology during 2005 to 2008, the IEEE Signal Processing Letters during 2003 to 2007, and the EURASIP Journal of Applied Signal Processing during 2001 to 2004. Dr. Xia served as a Member of the Signal Processing for Communications Committee from 2000 to 2005 and a Member of the Sensor Array and Multichannel (SAM) Technical Committee from 2004 to 2009 in the IEEE Signal Processing Society. Dr. Xia is Technical Program Chair of the Signal Processing Symp., Globecom 2007 in Washington D.C. and the General Co-Chair of ICASSP 2005 in Philadelphia. 